\title{
On Convergence of Development Costs and Cost Models for Complex Spaceflight Instrument Electronics
}

\author{
Semion Kizhner, Umeshkumar D. Patel, Robert L. Kasa, Phyllis Hestnes and Tammy Brown \\ National Aeronautics and Space Administration Goddard Space Flight Center \\ Semion.Kizhner-1@nasa.gov \\ Meg Vootukuru \\ Syneren Technologies Corporation \\ meg@syneren.com
}

\begin{abstract}
Development costs of a few recent spaceflight instrument electrical and electronics subsystems have diverged from respective heritage cost model predictions. The cost models used are Grass Roots, Price-H and Parametric Model. These cost models originated in the military and industry around 1970 and were successfully adopted and patched by NASA on a mission-by-mission basis for years. However, the complexity of new instruments recently changed rapidly by orders of magnitude. This is most obvious in the complexity of representative spaceflight instrument electronics' data system. It is now required to perform intermediate processing of digitized data apart from conventional processing of science phenomenon signals from multiple detectors. This involves on-board instrument formatting of computational operands from row data for example, images), multi-million operations per second on large volumes of data in reconfigurable hardware (in addition to processing on a general purpose imbedded or standalone instrument flight computer), as well as making decisions for on-board system adaptation and resource reconfiguration. The instrument data system is now tasked to perform more functions, such as forming packets and instrument-level data compression of more than one data stream, which are traditionally performed by the spacecraft command and data handling system. It is furthermore required that the electronics box for new complex instruments is developed for one-digit watt power consumption, small size and that it is light-weight, and delivers super-computing capabilities. The conflict between the actual development cost of newer complex instruments and its electronics components' heritage cost model predictions seems to be irreconcilable. This conflict and an approach to its resolution are addressed in this paper by determining the complexity parameters,
\end{abstract}

complexity index, and their use in enhanced cost model.

\subsection{Introduction}

The representative complex spaceflight instruments and prototypes are, for example, the Ocean Carbon Ecosystem and Near Shore Processes Mission (OCEaNS) optical instrument with high volume and high rate data streams from its focal planes and requiring on-board digital time delay integration. The non-optical Magnetospheric MultiScale Fast Plasma Investigation (MMS/FPI) instruments require on-board intensive computation of the Burst Quality Index. The Laser Interferometer Space Antenna (LISA) may require quad precision floating-point arithmetic computations and the Solar Viewing Interferometer Instrument Prototype (SVIP) is based on image processing within fast attitude control loops. A complex instrument is one that has large arrays of large detectors at fast readout rates, producing signal volumes and rates of order of magnitudes higher than heritage instruments and instrument based random access memory around 500 megabytes, and requiring non-trivial analog signal and digital data processing on-board the instrument.

There is a solution - to fly a super-computer, but obviously, it is not feasible. There is another solution at the other end of the spectrum of possible solutions. It is in utilizing reconfigurable hardware (RC) to achieve onboard super-computer power as a substitute for a supercomputer. However, this technologically possible solution is tempting, at the conceptual design phase, to make an assumption that this solution can be implemented by the heritage design methodologies of scaling up a heritage instrument design ("a bigger box" approach). This turns out to be a strong assumption and at the following design development and implementation phase results in merely a partial solution. In turn, a partial solution invariably requires re-designs during the development phase, which 
leads to divergence of development costs from cost model predictions.

In order to find the full design solution for a complex instrument at the outset conceptual level and cost the full design, the instrument complexity parameters must first be determined from the requirements and accounted for in the conceptual design. The complexity parameters and the conceptual design to these complexity parameters must then be included in the instrument cost model. Once the instrument complexity parameters are analyzed then the labor and hardware cost, required for the instrument design to these parameters (Section 8), can be estimated using heritage cost models or accounted for in the enhanced cost model and alleviate the cost divergence problem.

The elaboration of the complexity parameters in this paper in Sections 2-7 is straight- forward, including the framework for the proposed enhanced cost model that is using these complexity parameters and the complexity index derived from them, as well as the empirical cost factor based on the complexity index. The design of the Electrical and Electronics (EE) Subsystem to the complexity parameters at conceptual phase is presented in Section 8 .

\subsection{Complexity Parameters and the Enhanced Cost Model Methodology}

In order to develop an enhanced cost model for complex instruments, a few preliminary issues have to be discussed. Most subsystems within the newer complex instruments closely follow the typical heritage instrument. For example, their mechanical and thermal subsystems, except for change in dimensions for small satellites or large observatory, are similar to heritage ones. However, the requirements for the electronics' data subsystem have undergone radical changes and its complexity parameters must now be reflected in the cost model. The sources of complexity are described below and the sequence of the complexity parameters can be depicted as selected points on the guideline from the heritage cost models to the enhanced cost model (Fig. 4).

\subsection{Sources of Complexity Parameters}

The complexity parameters are derived based on the detector analog signal and on-board digital data processing flow from the source phenomena to the spacecraft solid-state recorder (SSR), with the emphasis on their information communications' aspects. The methodology we used in deriving the complexity parameters and the enhanced cost model can be described by enumerating the main points of the analysis followed by enhanced cost model framework synthesis. The onboard sources of analog signals and digital data are:
- External to spacecraft and instrument

- Science Phenomenon (measured by large arrays)

- External Calibration Sources (Analog distant star, sun, moon)

- Spacecraft Commands (Digital Data from Ground/Space Link)

- Global Positioning System (GPS) Position Data and Time Signals

- Internal to spacecraft and instrument

- Science Analog-to-Digital Conversion Output

- On-Board Intermediate Data Processing Results

- Pre-Processing i.e. frame formatting, packetization

- On-board Processing i.e. signal processing, time delay integration, data compression, time tagging and high performance computation

- Engineering

- Internal Calibration Sources

- Housekeeping Data Sources

\subsection{Methodology Developmental Steps}

The enhanced cost model methodology is derived using the following steps:

- Construct Informal Table of Signal and Data Sources

- Construct Formal Classification Matrix of Analog Signals, Digital Data Sources

- Determine Relation Between Source Signal Pixel and Detector Super-Pixel

- Map Signal Sources Onto Instrument Detectors and Describe On-Board Data Processing Complexity Parameters Origin

- Demonstrate Instrument Cost Model emergence from heritage cost to the enhanced cost model.

\subsection{Cost Models and Complexity Cost Distribution Outline}

The Heritage Cost Models (HCM) are denoted as $u$ and are based on the four primary parameters of two functions $f_{v}, f_{v r}$ representing an implementation labor and heritage hardware cost parameter $H$ and defined as

$$
\begin{gathered}
v=f_{v}(P, D) \\
v_{r}=f_{v r}\left(P_{r}, D_{r}\right) \\
u=f_{u}\left(f_{v}(P, D), f_{v r}\left(P_{r}, D_{r}\right), H\right)=f_{u}\left(P, D, P_{r}, D_{r}, H\right)
\end{gathered}
$$


where $P$ is the number of analog signals, $D$ is the number of digital signals, $P_{r}$ is sampling rate for analog signals, $D_{r}$ is sampling rate for digital data and $H$ is the heritage hardware cost parameter.

The enhanced cost model (ECM) function $U$ is derived based on the heritage cost model $u$ parameters and the derived complexity parameters of functions $V$ and $V_{r}$ implementation labor. The ECM function $U$ depends on design of the reconfigurable hardware $(R C)$ to the complexity parameters and complexity index $K$, derived from the instrument data system complexity parameters, as described in the following sections. The complexity parameter functions $V, V_{r}$ and $U$ are defined as

$$
\begin{aligned}
& V=\max (P, S, D, I, F, R, O, f, A, M) \\
& V_{r}=\max \left(P_{r}, S_{r}, D_{r}, I_{r}, F_{r}, R_{r}, O_{r}, f_{r}, A_{r}, M_{r}\right) \\
& U=f_{U}\left(V, V_{r}, R C, K\left(V, V_{r}\right)\right) \\
& =f_{U}\left(\begin{array}{l}
P, S, D, I, F, R, O, f, A, M, P_{r}, S_{r}, D_{r}, \\
I_{r}, F_{r}, R_{r}, O_{r}, A_{r}, M_{r}, R C
\end{array}\right)
\end{aligned}
$$

where $P, D, P_{r}$ and $D_{r}$ were described above, $S$ and $S_{r}$ are the size and rate of the detector super-pixels, $I$ and $I_{r}$ are intermediate data volume and rate, $F$ and $F_{r}$ are native pixel-frame size and rate for a required full readout of a sensor, $R$ and $R_{r}$ are compression reduced data volume and rate, $O$ and $O_{r}$ are the number of operations per second to be performed by on-board computation algorithms and data stream rates for the computational operands, $f$ is the software and firmware size, $A$ is the arithmetic complexity parameter and $M$ is the parameter reflecting the complexity of the simulation model. These parameters are derived below.

\subsection{Placement of Complexity Costs}

The placement of costs, induced by instrument electronics subsystem complexity index, $\mathrm{K}$, in the enhanced cost model is of most importance. It is not in scaling up the direct labor or hardware costs for a heritage design and implementation or its funding reserves in which the complexity parameters are not costed one by one. The placement of cost in the enhanced cost model should rather be in the labor and hardware costs for finding a full solution and design that includes all complexity parameters one by one. The cost should also account for labor and hardware needed for pre-optimizing the design for an instrument with the index of complexity $\mathrm{K}$ and for tuning a complex electronics subsystem at all phases along the design implementation path. In other words, the placement of the costs associated with the EE subsystem complexity occurs at all the phases of a project life cycle and can be accomplished in two ways:
- Complexity costs are manually converted by a user to costs in terms of labor and hardware inputs, when a heritage cost model is used; Even a heritage cost model $\mathbf{u}$ results in more accurate cost predictions, if all complexity parameters are taken into consideration at conceptual design phase

- In future work, one of the heritage cost models shall be modified to include complexity parameters in its database, yielding the enhanced cost model implementation as a new commercial tool.

In both case the cost of complexity parameters can be derived based on:

- Requirement Complexity Analysis

- Complexity risks assessment and mitigation by pre-proposal studies and $\mathrm{RC}$ hardware simulations

- Finding a solution at conceptual design phase

- Synthesis of solution, including RC algorithms pre-hardware optimization

\subsection{Implementation Steps}

- Instrument Data Processing Unit (IDPU) complexity implementation options are analyzed

- Design of a complex instrument to the complexity parameters is implemented at conceptual level and costed using the enhanced cost model framework.

\subsection{Sources of Instrument Analog Signals and Digital Data}

Heritage instruments were "throughput systems" from an instrument focal plane sensor assembly (FPA) source to destination - the spacecraft computer system and its solidstate recorder (SSR), in relation to the analog signal and digital data communications aspect. For example, a heritage instrument, such as the SeaStar spacecraft WideField-of-view Sensor (SeaWiFS) instrument comprises a few sensors with a small number of homogeneous source pixels - a source of a small number of analog signals $\mathbf{P}$ that are sampled at moderate sampling rate $\mathbf{P}_{\mathbf{r}}$. After digitization of $\mathbf{P}$ by the instrument, the digital data is the instrument output at data volume and rate $\left(\mathbf{D}, \mathbf{D}_{\mathbf{r}}\right)$. The digital data is then just forwarded to the spacecraft for 
processing and compression by one of the spacecraft computers and storage in the spacecraft SSR. The digital data volume essentially amounts to the volume of source pixels $\mathbf{P}$ multiplied by the analog-to-digital conversion (ADC) word length (usually $\Rightarrow 10$ to 16 bits), while the digital data rate in words is the same as the rate of source pixel signal sampling. Exception among heritage instruments was a large Observatory with complexity handled by costly Application Specific Integrated Circuits (ASIC). However, the large observatories, long-term schedules and multi-million dollars ASICs are not a common option today.

Representative advanced instruments are much more complex with strict cost and schedule constraints. These instruments study multiple non-homogeneous phenomena at different sampling rates and digitization resolution, and required to perform computationally intensive on-board digital data processing. For example, On-Board fast optics control loops require processing by the instrument of large CCD images $(512 \times 256$ pixels $)$ at hundreds of frames per second.

This requires a new look at the effects of different signal and digital data sources and their volumes and rates on the instrument power consumption and cost, in order to enhance the instrument electronics cost model at early stages of the instrument conceptual design, when instrument initial complexity and cost are evaluated at proposal and feasibility studies' levels.

The complexity parameters were not accounted for in heritage instrument cost models. The impact of complexity parameters for heritage instruments was so negligent, in comparison with that in newer instruments, that they could be reasonably ignored at conceptual level design.

For newer advanced instruments any omission of complexity parameters in costing the on-board data processing at the conceptual design or the Technical Management Review (TMR), or in the Final Proposal, could result in eventual cost underestimation and schedule overruns.

In order to derive complexity parameters for new instruments there is a need to derive a set of all signal sources and data in the form of a matrix. This matrix can be used in new complexity parameter derivation. The remainder of this section will explain in detail the signal and data source matrix derivation.

\subsection{External to Instrument and Spacecraft Signal Sources}

\subsubsection{Science Signal Sources}

The advanced subject instrument's science and engineering (calibration or housekeeping) signals originate from some physical phenomenon. The science data originates with measurements by the instrument detectors of the source signals' amplitude.

\subsubsection{External Calibration Sources}

To maintain the calibration system on-board, it is desirable to obtain reference calibration source measurements throughout the operational phase of a sensor. There are various calibration sources external to spacecraft being used for calibration system as: Distant Star, Sun, Moon, Orbit Night Sky or Spacecraft Dark Side, Earth Horizon and Bright Cloud.

\subsection{Internal or Engineering Signal Sources}

The engineering data originates within the instrument itself, namely the housekeeping detectors (thermal, pressure, force gouges, instrument or spacecraft power sources and other sensors) or instrument science detectors calibration sources, which are emulating natural physical phenomenon for the science physical phenomena detectors.

\subsubsection{Calibration Signal Sources}

Instrument-carried calibration sources - hot/cold plates, illumination spheres, etc.

\subsection{Instrument Digital Data Sources}

There is also some instrument digital data that originates in the instrument data (information) system apart from its detector signal ADCs (computation registers, intermediate digital data, telemetry format headers memory, SSR), and which needs to be transmitted to upper nodes of the spacecraft for communication to the ground control station or other spacecraft in a constellation. The physical phenomena itself can be comprised of electromagnetic radiation in different spectral ranges (ultra-violet, visible light, near Infrared or IR, thermal IR, communications radio-frequency or RF waves), mechanical forces, gravitation, chemical processes. These are also termed analog signals and, in turn, may have as their sources the sun, moon, a star, celestial background radiation or surface of planets - earth land and oceans' upward irradiation. Communications RF sources are, for example - commands, up-linked from a terrestrial or space control center to spacecraft and instrument.

\subsection{Informal Signal and Data Sources Classification Table}

Pictorially the set of all instrument analog signal and digital data sources, which contribute to instrument information, can be represented in an informal Table, Figure 1.

The informal multi-classification of instrument analog signal and digital data sources in Figure 1 can be further formalized into extended matrix as depicted in Figure 2. 


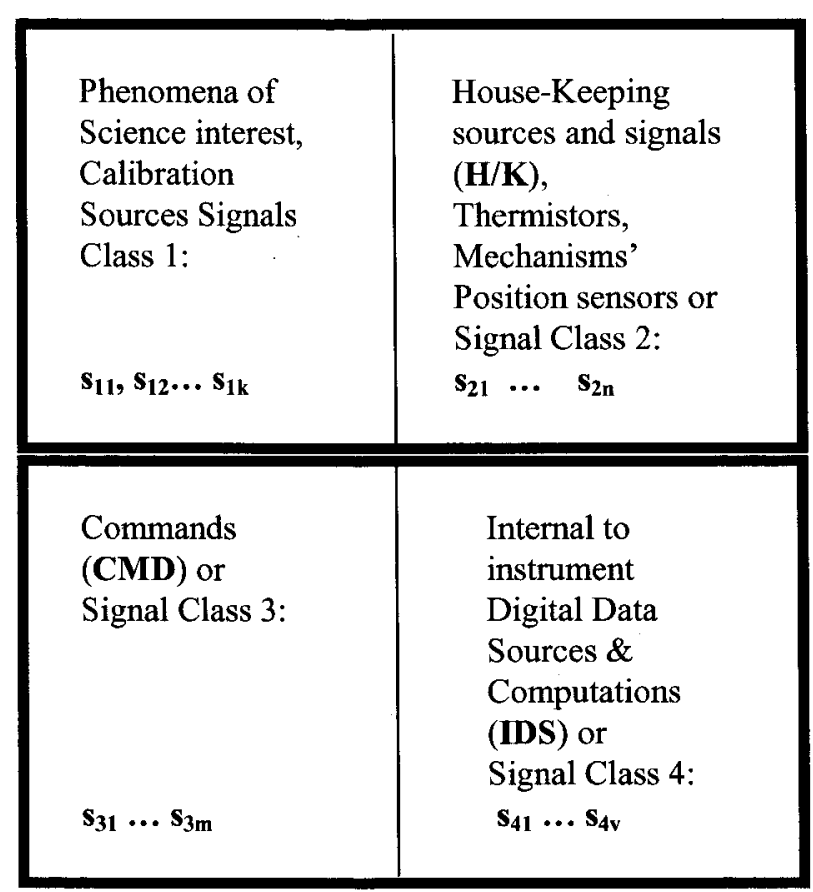

Figure 1. Classification of Instrument Analog Signals and Digital Data Sources

\subsection{Formal Classification of Sources}

The source with most elements $(\mathrm{H} / \mathrm{K}$ is perhaps the one, because it contains dozens of thermistors) is

$$
\left\{s_{21} \ldots s_{2 n}\right\}
$$

It comprises the class of the longest non-zero energy source of signals. Other sources for other analog signals and digital data are extended by zero-value elements to yield a sparsely populated " $4 \mathrm{x} \mathrm{n"} \mathrm{rectangular} \mathrm{extended}$ matrix of instrument data sources.

$$
\begin{array}{llll}
\mathrm{s}_{11} \mathrm{~s}_{12} \ldots \mathrm{s}_{1 \mathrm{k}} 0_{1 \mathrm{k}+1} \ldots & & 0_{1 \mathrm{n}} \\
\mathrm{s}_{21} \mathrm{~s}_{22} \ldots & & \mathrm{s}_{2 \mathrm{n}-1} & \mathrm{~s}_{2 \mathrm{n}} \\
\mathrm{s}_{31} \ldots \mathrm{s}_{3 \mathrm{~m}} 0_{3 \mathrm{n}+1} & \ldots & & 0_{3 n} \\
\mathrm{~s}_{41} \ldots & \mathrm{s}_{4 \mathrm{v}} 04 \mathrm{v}_{+1} \ldots & 0_{4 n}
\end{array}
$$

Figure 2. Extended Matrix of Instrument Data Sources

This formal classification matrix is used in the following sections to derive the new instruments complexity parameters, which are, in turn, used to develop the enhanced cost model for the electronics subsystem.

\subsection{Mapping Signal Sources onto Instrument Detectors}

We will examine now the propagation of the signal and data elements from the formal matrix of sources (Figure 2) throughout an instrument and associated complexity parameters.

A physical phenomenon manifests itself in emanated energy from some finite size (for example, $1 \mathrm{~km} \mathrm{x} 1 \mathrm{~km}$ ) surface or volume region - source signal pixel and this energy is being intercepted by an instrument detector, as a sub-pixel, pixel or super-pixel (binning of a few adjacent CCD pixels, for example (Figure 3 ).

\subsection{On Source Signal Pixels and Detector Super-Pixels}

A Source is usually associated with some finite size geometric space, say a terrestrial 2-D surface or 3-D volume (source pixel), or a small spot on an instrument surface for mounting a thermistor - a temperature signal detector as listed in Figure 1 above. For example, consider two sources:

A ground track of dimensions $\mathrm{L} x \mathrm{~h}$ with a Ground pixel of size $h \times h$ and

A spot on an instrument surface of radius $r$ for mounting a thermistor

Stylized Swath of L Source Pixels

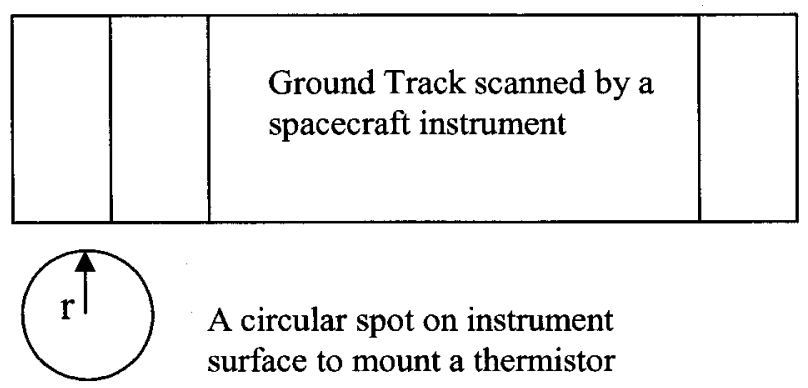

Figure 3. Geometry of Source Pixels

The number $\mathbf{P}$ of source pixels for the instrument is the sum of all non-zero elements in the above matrix $M$ :

$$
\mathbf{P}=\Sigma\left(\mathbf{s}_{\mathrm{ij}}\right)
$$

\subsection{On Mapping Signal Sources onto Detectors and Complexity Parameters}

Instrument sensors invariably comprise a set of detectors or sensing pixels out of which a super-pixel $d_{i j}$ of size $\mathbf{Z}$ (say, $Z=3 \times 3$ ) is comprised. For example, a CCD comprises a 2-D array of ( $\mathrm{n} \times \mathrm{m})$ sensing pixels that can be organized into a set of super-pixels on the CCD itself. An InGaAs linear array comprises a linear array of $(1 \times \mathrm{k})$ pixels and a housekeeping thermistor sensor usually comprises a single pixel thermal detector.

Each signal source $s_{i j}$ is further defined by its measurement interval $t_{i j}$ in fractions of a second (or sampling rate derived from this time as its inverse with frequency in $\mathrm{Hz}$ units). 
Although the sampling of different sources may not be asynchronous, the source sampling interval provides the definitive answer on the information volume of a signal source from communication point of view - in terms of source measured pixels per second.

It is obvious that at some time $\mathbf{T}$ the different samplings may intersect in time and that the instrument must be designed to handle the maximum combined data volume rate of

$$
\mathbf{P}_{\mathbf{r}}=\sum\left(\mathbf{s}_{\mathrm{ij}} / \mathbf{t}_{\mathrm{ij}}\right)
$$

pixels per second for all $\mathrm{i}, \mathrm{j}$; the division is 0 for $\mathrm{s}_{\mathrm{ij}}=0$.

$\mathbf{P}$ and $\mathbf{P}_{\mathbf{r}}$, the volume of the instrument observed source pixels and sampling rates handled by the instrument are the two important first parameters to consider in the evaluation of the instrument complexity and cost or $\mathbf{I}_{\mathbf{e c}}$ :

$$
\mathbf{I}_{\mathrm{cc}}=\mathbf{f}\left(\left\{\mathbf{P}, \mathbf{P}_{\mathrm{r}}\right\} \ldots\right)
$$

Next, the energy of a group of adjacent source pixels $s_{i j}$ energy is intercepted by an optical system and concentrated on a so-called Time Delay Integration slit of length determined by the TDI-size. This slit length L (in adjacent source pixels) is mapped into a TDI-size $=\mathrm{L}$ detector's spectrometer and each TDI input pixel is distributed over a column of detector $L$ super-pixels $d_{i j}$.

A source pixel's energy may further be practically instantaneously distributed in some spectral band over a $\lambda$ row of $d_{i j \lambda}$ detector super-pixels (CCD row) by different optical means, such as diffraction gratings.

This mapping of physical phenomena source pixels $s_{i j}$ onto instrument detector pixels results in the next level of data complexity, the number of detector super-pixels $\mathbf{S}$, namely

$$
S=\left(\sum\left(d_{i j}\right)\right) / Z>=L \times \lambda
$$

It is reasonable to evaluate the detector pixels' sampling rate by above $\mathbf{d}_{\mathrm{ij \lambda}}$ and say that

$$
\mathbf{S}_{\mathrm{r}}=\sum\left(\mathbf{d}_{\mathrm{ij} \lambda} / \mathbf{t}_{\mathrm{ij}}\right)
$$

The number $\mathbf{S}$ may be larger than $\mathbf{P}$ by an order of two magnitudes and it becomes the next important parameter in figuring out the Icc, namely:

1 source pixel is mapped onto detector $\lambda$ spectral superpixels on one CCD row per sampling time $t_{\mathrm{ij}}$.

The next level of instrument complexity stems from the detector super-pixel digitization into a fixed number of information bits $b\left(d_{i j \lambda}\right)$. This internal to the instrument data source is the detector source super-pixel analog-to-digital signal's converter $\mathrm{ADC}_{\mathrm{ij} \lambda}$, resulting in a detector pixel digital data volume and corresponding digital data rate from analog and $A D C$ board to the instrument digital information processing board or instrument data processing unit (IDPU):

$$
\begin{aligned}
& D=\sum\left(\mathbf{d}_{\mathrm{ij} \lambda} \mathbf{x} \mathbf{b}\left(\mathbf{d}_{\mathrm{ij} \lambda}\right)\right) \\
& \mathrm{D}_{\mathrm{r}}=\sum\left(\mathbf{d}_{\mathrm{ij} \lambda} \mathbf{x} \mathbf{b}\left(\mathbf{d}_{\mathrm{ij} \lambda}\right) \times \mathbf{t}_{\mathrm{ij}}\right)
\end{aligned}
$$

$\mathrm{D}$ and $\mathrm{D}_{\mathrm{r}}$ are the instrument internal digital data volumes and rates that may be orders of magnitude higher than then the previous level parameters $S$ and $S_{r}$, which are related to analog data sources. This is because the digitization width $b\left(d_{i j \lambda}\right) \Rightarrow W=>14$ bits within advanced instruments of today and tomorrow and it further contributes to Icc:

$\mathbf{I c c}=\mathbf{f}\left(\left\{\mathbf{P}, \mathbf{P}_{\mathrm{r}}, \mathbf{t}_{\mathrm{ij}}\right\},\left\{\left(\mathrm{d}_{\mathrm{ij}}, \mathbf{Z}\right), \boldsymbol{\lambda}, \mathbf{S}, \mathbf{S}_{\mathrm{r}}\right\},\left\{\mathbf{W}, \mathbf{D}, \mathbf{D}_{\mathrm{r}}\right\} \ldots\right)$

Next comes the on-board processing of the intermediate digital information within the instrument which involves transforming $\mathrm{D}, \mathrm{D}_{\mathrm{r}}$ into Intermediate data $\mathrm{I}$ and Intermediate rates $I_{r}$ that may be larger than $D$ and $D_{r}$ by a factor of $\mathrm{L}=2$ or more. For example, converting the $\mathrm{W}=14$-bit $\mathrm{ADC}$ counters into a 32-bit integer or single precision floating-point numbers, doubles $(32 / 14=$ factor $\mathrm{L}>2$ ) the volume and rate of $\mathrm{ADC}$ digital data to be processed within an IDPU or

$$
\begin{gathered}
I=2 \times D \\
I_{r}=2 \times D_{r}
\end{gathered}
$$

On-board post-ADC digital data Interpolation within an instrument IDPU (required in running some on-board algorithms) and data paketization overhead may increase the data volume and rate by a factor of $L>10$. Digital domain Time Delay Integration (TDI) may, in turn, decrease the intermediate data volume and rates at the price of required computational resources to implement the TDI algorithms. This further contributes to the Icc: $I c c=f\left(\left\{P, P_{r}, t_{i j}\right\},\left\{d_{i j \lambda}, Z, S, S_{r}\right\},\left\{W, D, D_{r}\right\},\left\{I, I_{r}, L\right.\right.$, TDI $\}$...)

Instrument detector calibration and fault detection may require, for example, to dump the entire CCD or an IDPU memory region. This requires the instrument to be able to handle data rates determined by native pixel-frame size and rate of full dump, say $\mathbf{F}, \mathbf{F}_{\mathrm{r}}$ or

$$
\begin{aligned}
& I c c=f\left(\left\{P, P_{r}, t_{i j}\right\},\left\{d_{i j \lambda}, Z, S, S_{r}\right\},\left\{W, D, D_{r}\right\},\left\{I, I_{r} ; L,\right.\right. \\
& \text { TDI }\left\{\mathbf{F}, \mathrm{F}_{\mathrm{r}}\right\} \ldots \text { ) }
\end{aligned}
$$

Furthermore, instrument-level data volume Reduction using Compression techniques by a pre-determined minimum factor (for example 2:1 lossless compression) may also reduce the data volume and rate to $R$, Rr. It bears on instrument complexity and cost:

$$
\begin{aligned}
& I c c=f\left(\left\{P, P_{r}, t_{i j}\right\},\left\{d_{i j \lambda}, Z, S, S_{r}\right\},\left\{W, D, D_{r}\right\},\left\{I, I_{r} ; L,\right.\right. \\
& \text { TDI } \left.\},\left\{F, F_{r}\right\},\left\{R, R_{r}\right\} \ldots\right)
\end{aligned}
$$

\subsection{On-Board Computational Complexity}

There are several flight missions where processors have been used that demonstrates the on-board heritage computational resource:

- FAST $10 \mathrm{MHz}$ general-purpose processor - SeaStar $16 \mathrm{MHz} 3$ primary, 3 backup microprocessors - TRMM $133 \mathrm{MHz}$ general-purpose processor. 
The heritage instruments, as exemplified by the instruments on spacecraft missions TRMM, SeaStar and FAST, could be characterized as

Class (I):

- High data volumes and rates

- Electronics Box is essentially through-putting high rate data to spacecraft (SC) Solid State Recorder (SSR)

- Low volume data is processed by the instrument data system computer

- These instruments require only moderate computational performance software-centric and their software aspect is preeminent.

Advanced complex instruments, such as the MMS/FPI instruments require more than $\mathrm{O}=40$ millions of computational operations per second on more than $\mathbf{O}_{\mathbf{r}}=$ 10 million bits per second data streams in single and double precision floating point arithmetic. Complex instruments can be characterized as $\mathrm{RC}$-Hardware-Centric Data Processing Instruments or

\section{Class (II):}

- High data volumes and rates

- Electronics Box is performing on-board high intensive large volume data processing in $\mathrm{RC}$ hardware as opposed to heritage ASIC

- The low volume housekeeping data is processed by the instrument's data system general-purpose computer

- These instruments are high computational performance $R C$-Hardware-Centric data processing instruments and their software computational aspect is minimal, while instrument flight software control aspect is still vital.

It is obvious from laboratory simulations that by only changing the type of variables from single precision to double precision, increases a small application's run time by a factor of. Changing data types 2 and to quad precision increases the run time by an order of magnitude. This is to simply demonstrate that on-board computations within an IDPU for an advanced instrument are not possible with heritage resources and requires FPGA implementations supplemented by host processors. In Class (II) instruments the Hardware (H/W) aspect of the Data System is the driver and the Software (S/W) aspect is minimal. This must be reflected in resource allocation, where

\section{S/W FTE $<<$ H/W FTE}

Because of this the computational complexity parameters $\mathbf{O}$ and $\boldsymbol{O}_{\mathrm{r}}$ (where often $\mathbf{O}_{\mathrm{r}}=\mathbf{D}_{\mathbf{r}}$ ) must be included in the instrument IDPU conceptual design and cost model:

$I c c=f\left(\left\{P, P_{r}, t_{i j}\right\},\left\{d_{i j \lambda}, Z, S, S_{r}\right\},\left\{W, D, D_{r}\right\},\left\{I, I_{r} ; L\right.\right.$, $\left.\mathrm{TDI}\},\left\{\mathbf{F}, \mathrm{F}_{\mathbf{r}}\right\},\left\{R, \mathbf{R}_{\mathrm{r}}\right\},\left\{\mathrm{O}, \mathrm{O}_{\mathrm{r}}\right\}\right)$

\subsection{Instrument Flight Software for a Constellation}

The Instrument Flight Software $f$ or Firmware - be to processor software or FPGA VHDL Code significantly affects the cost model. The flight software complexity affecting the model is designated as $\mathbf{f}$ or $\mathbf{f} / \mathbf{m}$, where $\mathbf{m}$ is the number of homogeneous spacecrafts/instruments in the constellation configured with the same instrument software with the S/W development costs counted only once. The software size $f$ also bears on model cost. The cost model then includes the associated $\mathrm{S} / \mathrm{W}$ parameter $\mathrm{f}$ :

$$
\begin{gathered}
I c c=f\left(\left\{P, P_{r}, t_{i j}\right\},\left\{d_{i j \lambda}, Z, S, S_{r}\right\},\left\{W, D, D_{r}\right\},\left\{I, I_{r} ; L,\right.\right. \\
\text { TDI } \left.\},\left\{F, F_{r}\right\},\left\{R, R_{r}\right\},\left\{O, O_{r}\right\}, f\right)
\end{gathered}
$$

\subsection{Computation Arithmetic}

Furthermore, the choice of arithmetic $\mathbf{A}$ - floating-point as opposed to fixed-point arithmetic affects

- Input Data Dynamic Range

- Allowable Depth of Computations before loosing precision

- Processing Time Depth.

In other words, the arithmetic choice parameter bears significant design costs, increasing with fixed-point, in particular and reflecting in the cost model:

$$
\begin{aligned}
& \mathbf{I c c}=\mathbf{f}\left(\left\{P, P_{\mathrm{r}}, \mathrm{t}_{\mathrm{ij}}\right\},\left\{\mathbf{d}_{\mathrm{ij} \lambda}, \mathbf{Z}, \mathbf{S}, \mathrm{S}_{\mathrm{r}}\right\},\left\{\mathbf{W}, \mathbf{D}, \mathrm{D}_{\mathrm{r}}\right\},\left\{\mathbf{I}, \mathbf{I}_{\mathrm{r}} ; \mathbf{L},\right.\right. \\
& \text { TDI } \left.\},\left\{F, F_{r}\right\},\left\{R, R_{r}\right\},\left\{O, O_{r}\right\},\{f\},\left\{A, A_{r}\right\}\right)
\end{aligned}
$$

\subsection{Calibration Sources Complexity}

And finally, the calibration sources complexity must be taken into account by introducing parameter $\mathbf{M}$ for modeling the science phenomena data sources. This completes the new enhanced cost model framework:

$$
\begin{array}{r}
\text { Icc }=\mathbf{f}\left(\left\{P, P_{r}, t_{i j}\right\},\left\{d_{i j \lambda}, Z, S, S_{r}\right\},\left\{W, D, D_{r}\right\},\left\{I, I_{r} ; L,\right.\right. \\
\text { TDI } \left.\},\left\{F, F_{r}\right\},\left\{R, R_{r}\right\},\left\{O, O_{r}\right\},\{f\},\left\{A, A_{r}\right\}, M\right)
\end{array}
$$

\subsection{Enhanced Cost Model Using Complexity Index}

The heritage instrument performance model in use for heritage instrument cost evaluation is based on throughput parameters $\mathbf{P}, \mathbf{D}, \mathbf{P}_{\mathrm{r}}, \mathbf{D}_{\mathrm{r}}$ and heritage hardware designs $\mathbf{H}$ :

$$
\begin{gathered}
\mathbf{v}=\mathbf{v}(\mathbf{P}, \mathbf{D}) \\
\mathbf{v}_{\mathbf{r}}=\mathbf{v}_{\mathbf{r}}\left(\mathbf{P}_{\mathbf{r}}, \mathbf{D}_{\mathbf{r}}\right) \\
\mathbf{u}=\mathbf{u}\left(\mathbf{v}, \mathbf{v}_{\mathbf{r}}, \mathbf{H}\right)=\mathbf{u}\left(\mathbf{P}, \mathbf{D}, \mathbf{P}_{\mathbf{r}}, \mathbf{D}_{\mathbf{r}}, \mathbf{H}\right)
\end{gathered}
$$

A complex instrument data system must be designed to handle the largest data volume and data rates determined by all parameters and by employing new solutions in reconfigurable hardware (RC). The enhanced cost model depends on the system complexity index $\mathbf{K}$ derived from the complexity parameters: 


$$
\begin{gathered}
\mathbf{V}=\max (\mathbf{P}, \mathbf{S}, \mathbf{D}, \mathbf{I}, \mathbf{F}, \mathbf{R}, \mathbf{O}, \mathbf{f}, \mathbf{A}, \mathbf{M}) \\
\mathbf{V}_{\mathbf{r}}=\max \left(\mathbf{P}_{\mathbf{r}}, \mathbf{S}_{\mathbf{r}}, \mathbf{D}_{\mathbf{r}}, \mathbf{I}_{\mathbf{r}}, \mathbf{F}_{\mathbf{r}}, \mathbf{R}_{\mathbf{r}}, \mathbf{O}_{\mathbf{r}}, \mathbf{A}_{\mathbf{r}}, \mathbf{M}_{\mathbf{r}}\right) \\
\mathbf{U}=\mathbf{U}\left(\mathbf{V}, \mathbf{V}_{\mathbf{r}}, \mathbf{R C}, \mathbf{K}\right)=\mathbf{U}(\mathbf{P}, \mathbf{S}, \mathbf{D}, \mathbf{I}, \mathbf{F}, \mathbf{R}, \mathbf{O}, \mathbf{f}, \mathbf{A}, \\
\left.\mathbf{M}, \mathbf{P}_{\mathbf{r}}, \mathbf{S}_{\mathbf{r}}, \mathbf{D}_{\mathbf{r}}, \mathbf{I}_{\mathbf{r}}, \mathbf{F}_{\mathbf{r}}, \mathbf{R}_{\mathbf{r}}, \mathbf{O}_{\mathbf{r}}, \mathbf{A}_{\mathbf{r}}, \mathbf{M}_{\mathbf{r}}, \mathbf{R C}\right)
\end{gathered}
$$

It is not that the parameters were not in the considerations before. However, their affects were so negligent in comparison with $\mathrm{P}$ and $\mathrm{D}$ parameters, that they could be reasonably ignored at conceptual level design. When they were considerable, the heritage ASICs (Fig. 6) implementation came to the rescue sometimes accompanied by costly re-designs.

\subsection{Empirical Evaluation of the Enhanced Cost Model}

If heritage cost model $\mathrm{u}=\mathrm{u}\left(\mathrm{P}, \mathrm{P}_{\mathrm{r}}, \mathrm{D}, \mathrm{D}_{\mathrm{r}}, \mathrm{H}\right)$ resulting cost was $u=5 * \$ X$ (in some heritage cost unit, say millions of dollars) or $\$ 5 X$, where 5 was the number of parameters in the heritage cost model, namely the parameters $\mathrm{P}, \mathrm{D}, \mathrm{P}_{\mathrm{r}}$, $D_{r}$. The enhanced cost model empirical evaluation can be represented as

$$
\begin{gathered}
\mathbf{U}=\mathbf{U}\left(\mathbf{V}, \mathbf{V}_{\mathbf{r}}, \mathbf{R C}, \mathbf{K}\right) \sim \mathbf{u}\left(\mathrm{v}, \mathrm{v}_{\mathrm{r}}, \mathrm{H}\right)+\mathbf{K}\left(\mathbf{V}, \mathbf{V}_{\mathbf{r}}\right) \text { or } \\
\mathbf{U}=\mathbf{u}+\mathbf{\mathbf { k }}=\mathbf{u}+(\mathbf{\$ 4 . 0} \text { million dollars })
\end{gathered}
$$

The cost adjustment parameter $\mathbf{k}$ is an empirical evaluation of the complexity index

where $\mathbf{k}$ was derived as

$$
\mathbf{K}=\mathbf{K}\left(\mathbf{V}, \mathbf{V}_{\mathbf{r}}\right) \sim \mathbf{k}
$$

$$
\mathbf{K}\left(\mathbf{V}, \mathbf{V}_{\mathrm{r}}\right) \sim \mathbf{k}=\left(\left(\|\mathbf{V}\|+\left\|\mathbf{V}_{\mathbf{r}}\right\|\right) /\left(\|\mathbf{v}\|+\left\|\mathbf{v}_{\mathbf{r}}\right\|\right)\right)
$$

In this paper $\mathbf{k}=(20 / 5)=4.0$, where 20 is the number of complexity parameters in the enhanced cost model and 5 is the number of parameters in the heritage cost model. Factor $\mathbf{k}$ is also based on the experience gleaned from the development of a few recent missions, and expressed as the heritage cost model adjustment, in $\mathbf{k}$ millions of dollars $\left(\$ \mathbf{k} \times 10^{6}\right)$. Factor $\mathbf{k}$ can be refined in future work as complexity index $K=K\left(V, V_{r}\right)$ gets a better formulation and by design optimization outlined below in Section 8 .

The sequence of these complexity parameters comprises the nodes on the guiding line from the heritage cost model $\boldsymbol{u}$ to the new enhanced cost model $U$ (Fig. 4):

$$
\begin{aligned}
& \begin{array}{llllllllll}
\mathbf{P} & \mathrm{S} & \mathrm{D} & \mathrm{I} & \mathbf{F} & \mathrm{R} & \mathrm{O} & \mathrm{f} & \mathrm{A} & \mathrm{M}
\end{array} \\
& \begin{array}{llllllllll}
P_{r} & S_{r} & D_{r} & I_{r} & F_{r} & R_{r} & O_{r} & A_{r} & M_{r} & R C
\end{array} \\
& 00000000000
\end{aligned}
$$

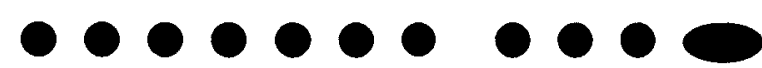

$$
\begin{aligned}
& U\left(V, V_{n} R C, K\right)
\end{aligned}
$$

$u\left(v, v_{r}, H\right)$

Figure 4. Guiding Line From Heritage Cost Model $u$ to the Enhanced Cost Model $U$

\subsection{The Enhanced Cost Model}

The enhanced cost model dependencies diagram presents the heritage inputs to the model, its functionality, as well as all the complexity parameters enumerated in this paper.

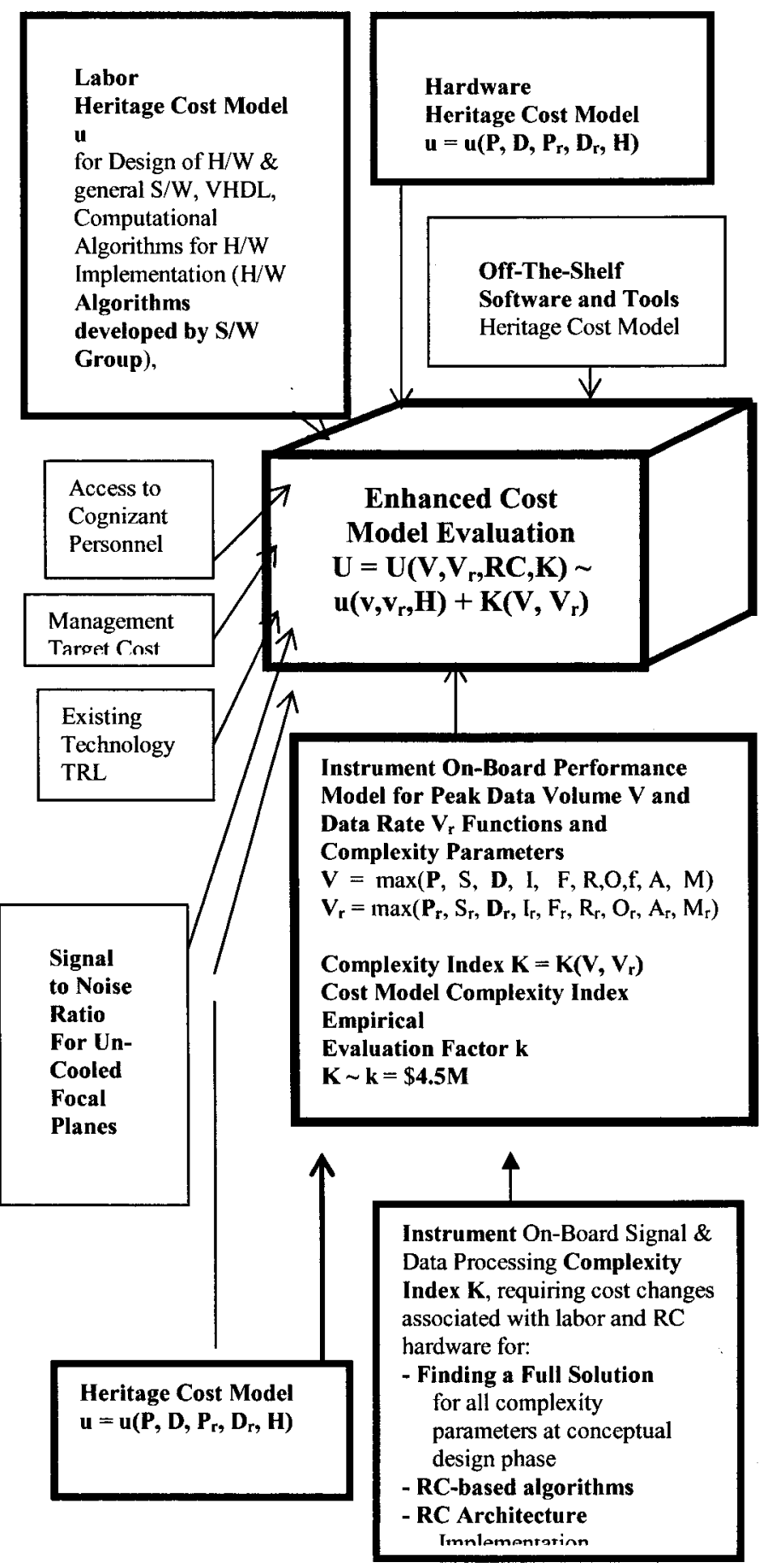

Figure 5. Enhanced Cost Model Dependencies 


\subsection{Implementation Options and Design to Complexity Parameters}

The cost for contemporary complex instruments discourage ASIC based design solutions. The current and future instruments are characterized by complexity parameters that are larger than $\mathbf{P}$ and $\mathbf{D}$ by orders of magnitude, resulting in the need to reconsider the IDPU complexity and cost models more explicitly. The resulting enhanced cost model is very different from the heritage cost model, as depicted in the Enhanced Cost Model Diagram in Figure 5. The new solutions for complex instruments are based on FPGA/RC technology implementations, which also allow solutions for a larger class of problems, but at a lower cost than with ASICs. Some implementation options for complex instruments are shown in a graphical form in Figure 6.

\subsection{Implementation Options}

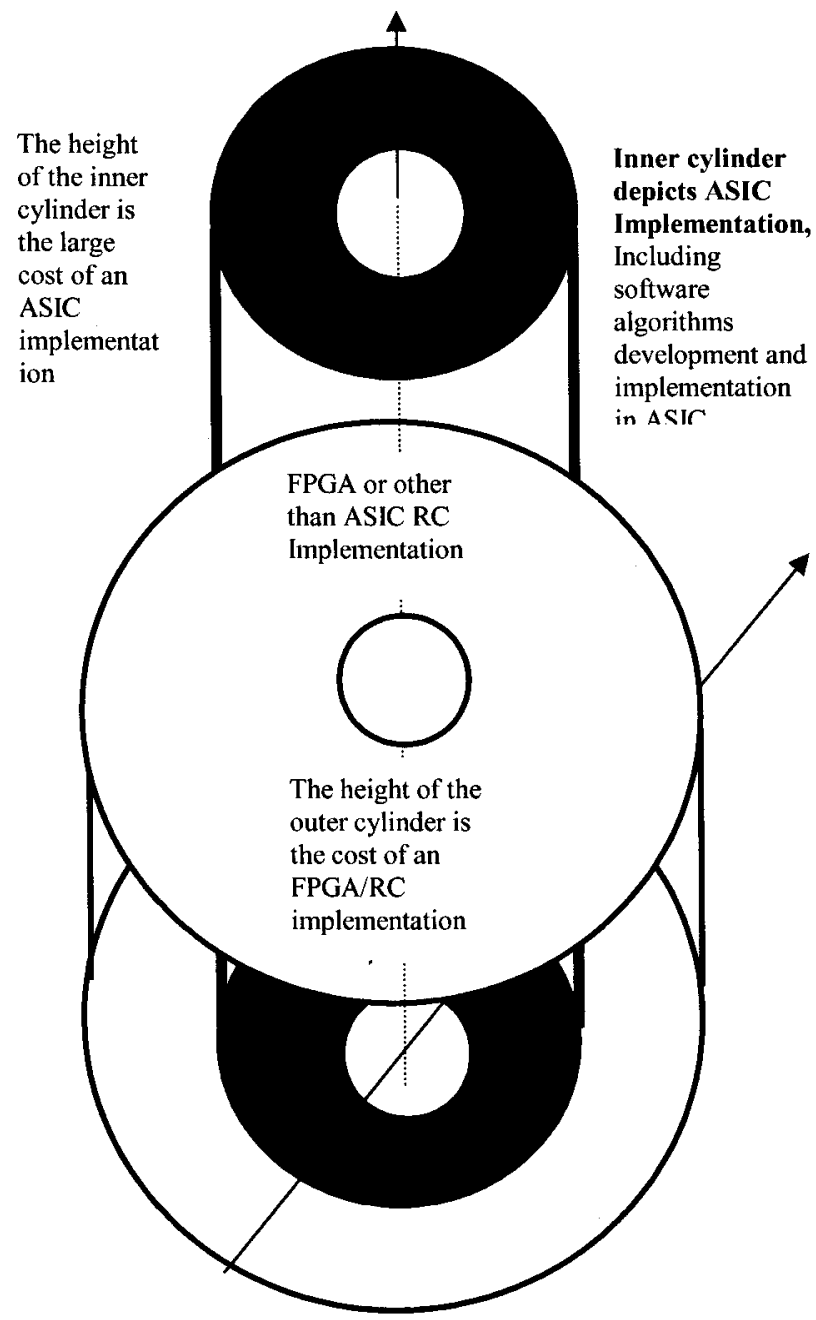

Figure 6. Comparative Costs of ASIC and FPGA Solution Implementations
In Picture 6 the inner white circle is an icon for a Complex Signal and Data Processing Application. Its encompassing solution is depicted in dark for an ASIC implementation and in white for an FPGA/RC implementation. Both solve larger problems at a cost.

\subsection{Design of an EE Subsystem to Complexity Parameters}

Contemporary complex instruments and their electrical and electronics data sub-systems must be designed from the conceptual outset to their complexity parameters and include these performance complexity parameters in the enhanced cost model $U$. The design to complexity parameters and its optimization may greatly affect the cost associated with the instrument complexity index $\mathbf{K}$, or even change its sign, resulting in overall cost reduction. For instance, an improvement of an on-board computational algorithm by a factor of 2 is huge because of the cost of on-board resources, while on the ground it is not worth the effort to achieve it. The design and optimization of an instrument on-board data system can, in turn, significantly affect the value and sign of the new cost model for the complexity index $\mathbf{K}$, reducing costs in other parts of the mission, like downlink bandwidth and ground processing.

For example, in instruments dealing with weak measurement signals, an on-board digital TDI computation is an algorithm of choice. The n-step TDI (TDI-n) algorithm is critical in improving the signal-tonoise ratio for weak Near Infrared (IR) and thermal band signals by a factor of sqrt(n). As a byproduct of TDI- $n$ process, the downlink data volume is decremented by a factor of $\mathbf{n}$. However, while source signal noise randomness accounts for averaging it out the analog signal digitization noise only adds up in lower bits of the digital counts and the lower two bits in the TDI output need to be discarded. The design to the intermediate parameter $I$ requires that the $\mathrm{ADC}$ is 2-bits wider than top-level requirements for the width of the downlink counters. On the other hand, the TDI computation of complexity $\boldsymbol{O}$ on this larger volume of intermediate data can be optimized by a factor of at least 2 by replacing the operation of division with multiplication by a pre-computed and stored in a small table of multiplicands $1 / j$, for $1<=j<=24$.

In any contemporary implementation of the operation of division the division takes 15-25 clock cycles, as compared to 3 cycles for subtraction, addition, and multiplication. When there is a short computation (like a TDI-6), involving five additions (+), and a single division (/), the division is much more lengthy in time cycles' duration than an entire TDI computation $\left(a_{1}+a_{2}+a_{3}+a_{4}\right.$ $\left.+a_{5}+a_{6}\right) / 6$, where $a^{j}$ are the detector pixels signal measurements for ground pixel $a$ at consecutive dwelling times $t_{j}$. 
However, when the dividers are known in advance and their number is small, for example in a TDI- $\mathrm{j}$ computation $(1<=j<=24)$, their inverse can be pre-computed and stored in a small table. Then, instead of division implementation in FPGA or its computer use, can be replaced by multiplication making the entire computation at least twice faster and coherent without any additional resources for an FPGA divider circuitry.

The design and development of the electronics data subsystem, based on the above derived complexity parameters, requires personnel labor and reconfigurable hardware, commensurate with the resulting correction factor k. Namely, it is empirically known that each million of dollars in cost is equivalent to 3 Full Time Equivalent developer labor units (FTEs). The cost attributed to complexity index $\mathrm{K}=5$ can be approximated by $\left(3^{*} \mathbf{k}\right)$ FTEs (approximately 15 FTEs) over the mission development period, in addition to the heritage cost which basically covers the materials, manufacturing and processor software, rather than the design to the complex parameters.

\section{Conclusions}

We characterized the class of representative complex instruments as Class II of RC-hardware-centric data processing instruments. In such instruments the complexity of on-board analog signal and digital data processing in reconfigurable hardware far supersedes that in the general-purpose heritage processors. For such instruments the EE subsystem development effort supersedes the heritage software-centric development methodology and an enhanced cost model must be applied to it. We have then analyzed the new representative complex instrument top-level requirements and associated data volumes, rates and on-board computational algorithms' RC implementations. We enumerated the EE subsystem data processing complexity parameters and functions $\mathrm{V}, \mathrm{V}_{\mathrm{r}}$ and introduced the instrument complexity index $\mathrm{K}\left(\mathrm{V}, \mathrm{V}_{\mathrm{r}}\right)$. We then introduced the complex instrument EE subsystem enhanced cost model framework $\mathrm{U}\left(\mathrm{V}, \mathrm{V}_{\mathrm{r}}, \mathrm{RC}, \mathrm{K}\right)$. Cost Model $\mathrm{U}$ is based on labor required to design for the complexity parameters and on cost of reconfigurable hardware. For complex instruments the EE subsystem must be designed to the complexity parameters one-by-one at the outset conceptual level. The costing must use the enhanced cost model $U$ that is based on the complexity parameters in order to avoid consecutive development costs' diversions and schedule overruns. The costs associated with the complexity parameters can be estimated in heritage ways, as long as the complexity parameters are costed at the outset conceptual level design and converted to labor and RC hardware inputs used by a heritage cost model. We derived the empirical evaluation of the enhanced cost model function as $\mathrm{U} \sim \mathbf{u}+\mathbf{k}(\mathrm{K})$.
Factor $\mathrm{k}(\mathrm{K})$ can also be viewed as the baseline cost of a complex instrument EE subsystem data. Future work consists in implementing the enhanced cost model framework into a cost engineering tool.

\section{Acknowledgements}

We would like to thank Paul Earle for constructive discussions of the paper.

\section{References}

[1] Model-H, Personal Communication from Paul Earle/NASA GSFC

[2] Cost Model for Electronics Architecture Trade Studies by J. Axelsson, Volvo Technological Corp., 2000 IEEE

[3] Parametric Cost Estimating by Ralph W. Farrington, RWF Consulting LLC, 2005 IEEE 


\title{
On Convergence of Development Costs and Cost Models for Complex Spaceflight Instrument Electronics
}

\author{
Semion Kizhner, Umeshkumar D. Patel, Robert L. Kasa, \\ Phyllis Hestnes and Tammy Brown \\ National Aeronautics and Space Administration \\ Goddard Space Flight Center \\ Greenbelt Road, Greenbelt MD, 20771 \\ 301-286-1294 \\ Semion.Kizhner-1@nasa.gov \\ Meg Vootukuru \\ Syneren Technologies Corporation \\ 703-738-6499 \\ meg@syneren.com
}

\begin{abstract}
Development costs of a few recent spaceflight instrument electrical and electronics subsystems have diverged from respective heritage cost model predictions. The cost models used are Grass Roots, Price-H and Parametric Model. These cost models originated in the military and industry around 1970 and were successfully adopted and patched by NASA on a mission-by-mission basis for years. However, the complexity of new instruments recently changed rapidly by orders of magnitude. This is most obvious in the complexity of representative spaceflight instrument electronics' data system. It is now required to perform intermediate processing of digitized data apart from conventional processing of science phenomenon signals from multiple detectors. This involves on-board instrument formatting of computational operands from row data (for example, images), multi-million operations per second on large volumes of data in reconfigurable hardware (in addition to processing on a general purpose imbedded or stand-alone instrument flight computer), as well as making decisions for on-board system adaptation and resource reconfiguration. The instrument data system is now tasked to perform more functions such as forming packets and instrument-level data compression of more than one data stream, which are traditionally performed, by the spacecraft command and data handling $(\mathrm{C} \& D H)$ system. It is furthermore required that the electronics box for new complex instruments is developed for one-digit watt power consumption, small size and that it is light-weight, and delivers super-computing capabilities. The conflict between the actual development cost of newer complex instruments and its electronics components' heritage cost model predictions seems to be irreconcilable. This conflict and an approach to its resolution are addressed in this paper by determining the complexity parameters, the complexity index, and the use of these in the enhanced cost model.
\end{abstract}




\section{Table of Contents}

On Convergence of Development Costs and Cost Models for Complex Spaceflight Instrument Electronics................................................................... 1

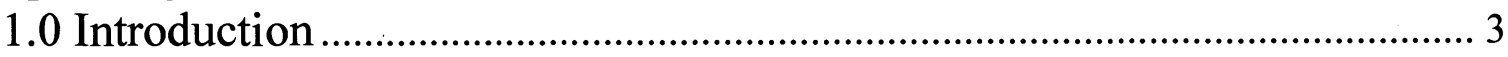

2.0 Complexity Parameters and the Enhanced Cost Model Methodology...... 4

2.1 Sources of Complexity Parameters............................................................................ 4

2.2 Methodology Developmental Steps ...................................................................... 4

2.3 Cost Models and Complexity Cost Distribution Outline ........................................... 5

2.3.1 Complexity Costs Placement ........................................................................ 5

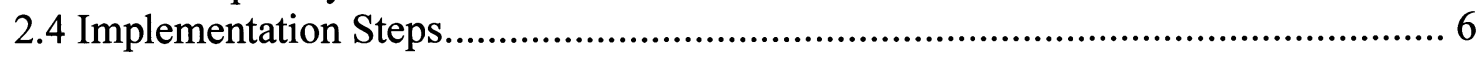

3.0 Sources of Instrument Analog Signals and Digital Data ............................. 6

3.1 External to Instrument and Spacecraft Signal Sources ........................................... 7

3.1.1 Science Signal Sources................................................................................... 7

3.1.2 External Calibration Sources ................................................................ 7

3.2 Internal or Engineering Signal Sources................................................................. 7

3.2.1 Internal House-Keeping Signal Sources....................................................... 8

3.2.2 Calibration Signal Sources ……………………......................................... 8

3.3 Instrument Digital Data Sources....................................................................... 8

3.4 Informal Signal and Data Sources Classification Table ......................................... 8

4.0 Formal Classification of Sources for Analog Signals and Digital Data... 9

5.0 Mapping Signal Sources Onto Instrument Detectors …………................... 9

5.1 On Source Signal Pixels and Detector Super-Pixels............................................... 9

5.2 On Mapping Signal Sources Onto Detectors and Complexity Parameters..............10

6.0 On-Board Computational Complexity .....................................................12

6.1 Instrument Flight Software for a Constellation ...................................................14

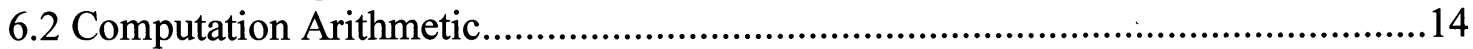

6.3 Calibration Sources Complexity ......................................................................

7.0 Enhanced Cost Model Using Complexity Index.............................................14

7.1 Empirical Evaluation of the Enhanced Cost Model................................................15

7.2. Enhanced Cost Model Diagram...........................................................................16

8.0 Design of the EE Subsystem to Complexity Parameters and Options .....17

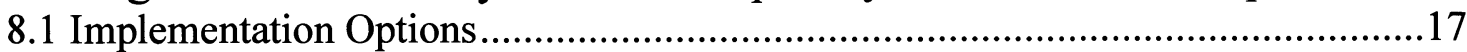

8.2 Design of an EE Subsystem to Complex Parameters ……....................................18

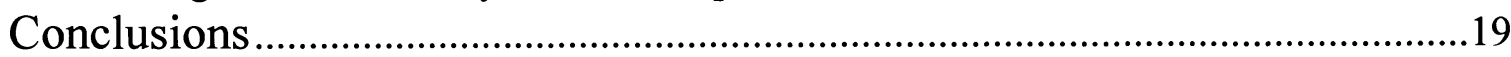

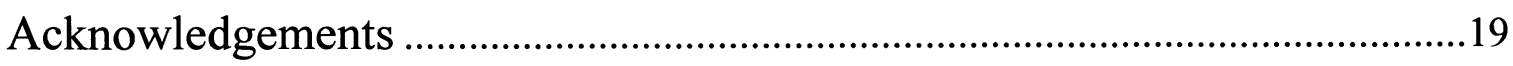

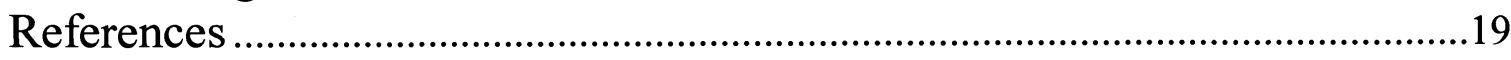

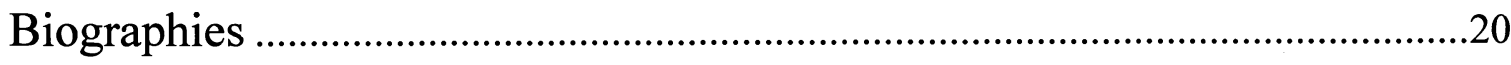




\subsection{Introduction}

The representative complex spaceflight instruments and prototypes are, for example, the Ocean Carbon Ecosystem and Near Shore Processes Mission (OCEaNS) optical instrument with high volume and high rate data streams from its focal planes and requiring on-board digital time delay integration. The non-optical Magnetospheric MultiScale Fast Plasma Investigation (MMS/FPI) instruments require on-board intensive computation of the Burst Quality Index. The Laser Interferometer Space Antenna (LISA) may require quad precision floating-point arithmetic computations and the Solar Viewing Interferometer Instrument Prototype (SVIP) is based on image processing within fast attitude control loops. A complex instrument is one that has large arrays of large detectors at fast readout rates, producing signal volumes and rates of order of magnitudes higher than heritage instruments and instrument based random access memory around 500 megabytes, and requiring non-trivial analog signal and digital data processing on-board the instrument.

There is a solution - to fly a super-computer, but obviously, it is not feasible. There is another solution at the other end of the spectrum of possible solutions. It is in utilizing reconfigurable hardware $(R C)$ to achieve on-board super-computer power as a substitute for a super-computer. However, this technologically possible solution is tempting, at the conceptual design phase, to make an assumption that this solution can be implemented by the heritage design methodologies of scaling up a heritage instrument design ("a bigger box" approach). This turns out to be a strong assumption and at the following design development and implementation phase results in merely a partial solution. In turn, a partial solution invariably requires re-designs during the development phase, which leads to divergence of development costs from cost model predictions.

In order to find the full design solution for a complex instrument at the outset conceptual level and cost the full design, the instrument complexity parameters must first be determined from the requirements and accounted for in the conceptual design. The complexity parameters and the conceptual design to these complexity parameters must then be included in the instrument cost model. Once the instrument complexity parameters are analyzed then the labor and hardware cost, required for the instrument design to these parameters (Section 8), can be estimated using heritage cost models or accounted for in the enhanced cost model and alleviate the cost divergence problem.

The elaboration of the complexity parameters in this paper in Sections 2-7 is straightforward, including the framework for the proposed enhanced cost model that is using these complexity parameters and the complexity index derived from them, as well as the empirical cost factor based on the complexity index. The design of the Electrical and Electronics (EE) Subsystem to the complexity parameters at conceptual phase is presented in Section 8. 


\subsection{Complexity Parameters and the Enhanced Cost Model Methodology}

In order to develop an enhanced cost model for complex instruments, a few preliminary issues have to be discussed. Most subsystems within the newer complex instruments closely follow the typical heritage instrument. For example, their mechanical and thermal subsystems, except for change in dimensions for small satellites or large observatory, are similar to heritage ones. However, the requirements for the electronics' data subsystem has undergone radical changes and its complexity parameters must now be reflected in the cost model. The sources of complexity are described below and the sequence of the complexity parameters can be depicted as selected points on the guideline from the heritage cost models to the enhanced cost model (Figure 4).

\subsection{Sources of Complexity Parameters}

The complexity parameters are derived based on the detector analog signal and on-board digital data processing flow from the source phenomena to the spacecraft solid-state recorder (SSR), with the emphasis on their information communications' aspects. The methodology we used in deriving the complexity parameters and the enhanced cost model can be described by enumerating the main points of the analysis followed by enhanced cost model framework synthesis. The on-board sources of analog signals and digital data are as follows:

- External to spacecraft and instrument

- Science Phenomenon (measured by large arrays)

- External Calibration Sources (Analog - distant star, sun, moon)

- Spacecraft Commands (Digital Data from Ground/Space Link)

- GPS Position Data and Time Signals

- Internal to spacecraft and instrument

- Science Analog-to-Digital Conversion Output

- On-Board Intermediate Data Processing Results

- Pre-Processing i.e. frame formatting, packetization

- On-board Processing i.e. signal processing, time delay integration, data compression, time tagging, high performance computation

- Engineering

○ Internal Calibration Sources

○ Housekeeping Data Sources

\subsection{Methodology Developmental Steps}

The enhanced cost model methodology is derived using the following steps:

- Informal Table of Signal and Data Sources

- Formal Classification Matrix of Sources for Analog Signals and Digital Data

- Source Signal Pixel and Detector Super-Pixel

- Mapping Signal Sources Onto Instrument Detectors and On-Board Data Processing Complexity Parameters Origin 
- Instrument Cost Model emergence from heritage cost to the enhanced cost model.

\subsection{Cost Models and Complexity Cost Distribution Outline}

The Heritage Cost Models (HCM) $\mathbf{u}$ are based on the four primary parameters of two functions $\mathbf{v}, \mathbf{v}_{\mathbf{r}}$ representing an implementation labor and heritage hardware cost parameter $\mathbf{H}$ described as:

$$
\begin{aligned}
& \text { Heritage Cost Model (HCM) } \\
& \qquad \begin{aligned}
\mathbf{v} & =\mathbf{f}(\mathbf{P}, \mathbf{D}) \\
\mathbf{v}_{\mathbf{r}} & =\mathbf{f}_{\mathbf{r}}\left(\mathbf{P}_{\mathbf{r}}, D_{\mathbf{r}}\right) \\
\mathbf{u} & =\mathbf{u}\left(\mathbf{f}(\mathbf{P}, \mathbf{D}), \mathbf{f}_{\mathbf{r}}\left(\mathbf{P}_{\mathbf{r}}, D_{\mathbf{r}}\right), \mathbf{H}\right)=\mathbf{u}\left(\mathbf{P}, \mathbf{D}, \mathbf{P}_{\mathbf{r}}, D_{\mathbf{r}}, \mathbf{H}\right)
\end{aligned}
\end{aligned}
$$

where $\mathrm{P}$ is the number of analog signals, $\mathrm{D}$ is the number of digital signals, $\mathrm{P}_{\mathrm{r}}$ is sampling rate for analog signals and $D_{r}$ is sampling rate for digital data.

The enhanced cost model $\boldsymbol{U}$ is based on the heritage cost model $\boldsymbol{u}$ parameters and the derived complexity parameters of functions $\boldsymbol{V}$ and $\boldsymbol{V}_{\boldsymbol{r}}$ implementation labor.

$\boldsymbol{U}$ depends on design of the reconfigurable hardware $(\boldsymbol{R C})$ to the complexity parameters and complexity index $\boldsymbol{K}$, derived from the instrument data system complexity parameters, as described in the following Sections.

$$
\begin{aligned}
& \text { Enhanced Cost Model (ECM) } \\
& \qquad \begin{array}{r}
\mathbf{V}=\max (\mathbf{P}, \mathbf{S}, \mathbf{D}, \mathbf{I}, \mathbf{F}, \mathbf{R}, \mathbf{O}, \mathbf{f}, \mathbf{A}, \mathbf{M}) \\
\mathbf{V}_{\mathbf{r}}=\max \left(\mathbf{P}_{\mathbf{r}}, \mathbf{S}_{\mathbf{r}}, \mathbf{D}_{\mathbf{r}}, \mathbf{I}_{\mathbf{r}}, \mathbf{F}_{\mathbf{r}}, \mathbf{R}_{\mathbf{r}}, \mathbf{O}_{\mathbf{r}}, \mathbf{A}_{\mathbf{r}}, \mathbf{M}_{\mathbf{r}}\right) \\
\mathbf{U}=\mathbf{U}\left(\mathbf{V}, \mathbf{V}_{\mathbf{r}}, \mathbf{R C}, \mathbf{K}\left(\mathbf{V}, \mathbf{V}_{\mathbf{r}}\right)\right)=\mathbf{U}(\mathbf{P}, \mathbf{S}, \mathbf{D}, \mathbf{I}, \mathbf{F}, \mathbf{R}, \mathbf{O}, \mathbf{f}, \mathbf{A}, \mathbf{M}, \\
\left.\quad \mathbf{P}_{\mathbf{r}}, \mathbf{S}_{\mathbf{r}}, \mathbf{D}_{\mathbf{r}}, \mathbf{I}_{\mathbf{r}}, \mathbf{F}_{\mathbf{r}}, \mathbf{R}_{\mathbf{r}}, \mathbf{O}_{\mathbf{r}}, \mathbf{A}_{\mathbf{r}}, \mathbf{M}_{\mathbf{r}}, \mathbf{R C}\right)
\end{array}
\end{aligned}
$$

where $P, D, P_{r}$ and $D_{r}$ are described above and the new parameters are derived below.

\subsubsection{Complexity Costs Placement}

The placement of costs, induced by instrument electronics subsystem complexity index $K$ in the enhanced cost model is of most importance. It is not in scaling up the direct labor or hardware costs for a heritage design and implementation or its funding reserves in which the complexity parameters are not costed one by one. The placement of cost in the enhanced cost model should rather be in the labor and hardware costs for finding a full solution and design that includes all complexity parameters one by one. The cost should also account for labor and hardware needed for pre-optimizing the design for an instrument with the index of complexity $\mathrm{K}$ and for tuning a complex electronics subsystem at all phases along the design implementation path.

In other words, the placement of the costs associated with the EE subsystem complexity occurs at all the phases of a project life cycle. This can be accomplished in two ways: 
1) Complexity costs are manually converted by a user to costs in labor and hardware inputs, when a heritage cost model is used; Even a heritage cost model $\boldsymbol{u}$ results in more accurate cost predictions, if it takes into consideration all complexity parameters at conceptual design phase (Figure 4)

2) In future work one of the heritage cost models shall be modified to include complexity parameters in its database, yielding the enhanced cost model implementation as a new commercial tool.

In both case the cost of complexity parameters placement is based on:

Analysis of the requirements' complexity

Complexity risks' assessment and mitigation by pre-proposal studies and $R C$ hardware experiments simulations

Finding a solution at conceptual design phase

Synthesis of solution, including RC algorithms' pre-hardware optimization.

\subsection{Implementation Steps}

- Instrument Data Processing Unit (IDPU) complexity implementation options are analyzed

- Design of a complex instrument to the complexity parameters is implemented at conceptual level and costed using the enhanced cost model framework.

\subsection{Sources of Instrument Analog Signals and Digital Data}

Heritage instruments were "throughput systems" from an instrument focal plane sensor source to destination - the spacecraft computer system and its solid-state recorder (SSR), in relation to the analog signal and digital data communications aspect. For example, a heritage instrument, such as the SeaStar spacecraft Wide-Field-of-view Sensor (SeaWiFS) instrument comprises a few sensors with a small number of homogeneous source pixels - a source of a small number of analog signals $\mathbf{P}$ that are sampled at moderate sampling rate $\mathbf{P}_{\mathbf{r}}$. After digitization of $\mathbf{P}$ by the instrument, the digital data is the instrument output at data volume and rate $\left(\mathbf{D}, \mathbf{D}_{\mathbf{r}}\right)$. The digital data is then just forwarded to the spacecraft for processing and compression by one of the spacecraft computers and storage in the spacecraft SSR. The digital data volume essentially amounts to the volume of source pixels $\mathbf{P}$ multiplied by the analog-to-digital conversion (ADC) word length (usually $=>10$ to 16 bits), while the digital data rate in words is the same as the rate of source pixel signal sampling. Exception among heritage instruments was a large Observatory with complexity handled by costly Application Specific Integrated Circuits (ASIC). However, the large observatories, long-term schedules and multi-million dollars ASICs are not a common option today.

Representative advanced instruments of today and tomorrow are much more complex with strict cost limitations and short schedules. These instruments study multiple nonhomogeneous phenomena at different sampling rates and digitization widths, as well as 
perform computationally intensive on-board digital data processing and compression within the instrument. For example, On-Board fast optics control loops require processing by the instrument of large CCD images (512 x 256 pixels) at hundreds of frames per second.

This, in turn, requires a new look at the affects of complex instrument's different signal and digital data sources and their volumes and rates on the instrument complexity (power consumption in particular) and cost. This is required in order to enhance the instrument electronics cost model at early stages of the instrument conceptual design, when instrument initial complexity and cost are evaluated at proposal and feasibility studies' levels.

It is not that such parameters were not accounted for in heritage instrument cost models. However, their impact so negligent, in comparison with that in newer instruments, that they could be reasonably ignored at conceptual level design.

For newer complex instruments any omissions in costing the complexity of on-board data processing at the early conceptual level design of a complex instrument or the Technical Management Review Package (TMR), or in a Final Research Proposal, could result in eventual project cost underestimation and schedule overruns.

In order to derive the new instrument's complexity parameters there is a need to take a closer informal look at the set of all the sources of signals and data and then formalize this set using the mathematical construct of a matrix. This matrix is then used to derive the new complexity parameters. This is done in the remainder of this Section and the following Section 3.

\subsection{External to Instrument and Spacecraft Signal Sources}

\subsubsection{Science Signal Sources}

The advanced subject instrument's science and engineering (calibration or housekeeping) signals originate from some physical phenomenon. The science data originates with measurements by the instrument detectors of the source signals' amplitude.

\subsubsection{External Calibration Sources}

Distant Star

Sun

Moon

Orbit Night Sky or Spacecraft Dark Side

Earth Horizon

Bright Cloud

\subsection{Internal or Engineering Signal Sources}

The engineering data originates with the instrument itself, namely within the housekeeping detectors (thermal, pressure, force gouges, instrument or spacecraft power 
sources and other sensors) or instrument science detectors calibration sources, which are emulating natural physical phenomenon for the science physical phenomena detectors.

\subsubsection{Internal House-Keeping Signal Sources}

Thermistors, Force sensors

\subsubsection{Calibration Signal Sources}

Instrument-carried calibration sources - hot/cold plates, illumination spheres, etc.

\subsection{Instrument Digital Data Sources}

There is also some instrument digital data that originates in the instrument data (information) system apart from its detector signal ADCs (computation registers, intermediate digital data, telemetry format headers memory, SSR), and which needs to be transmitted to upper nodes of the spacecraft for communication to the ground control station or other spacecraft in a constellation. The physical phenomena itself can be comprised of electromagnetic radiation in different spectral ranges (ultra-violet, visible light, near Infrared or IR, thermal IR, communications radio-frequency or RF waves), mechanical forces, gravitation, chemical processes. These are also termed analog signals and, in turn, may have as their sources the sun, moon, a star, celestial background radiation or surface of planets - earth land and oceans' upward irradiation. Communications RF sources are, for example-commands, up-linked from a terrestrial or space control center to spacecraft and instrument.

\subsection{Informal Signal and Data Sources Classification Table}

Pictorially the set of all instrument analog signal and digital data sources, which contribute to instrument information, can be represented in an informal Table, Figure 1.

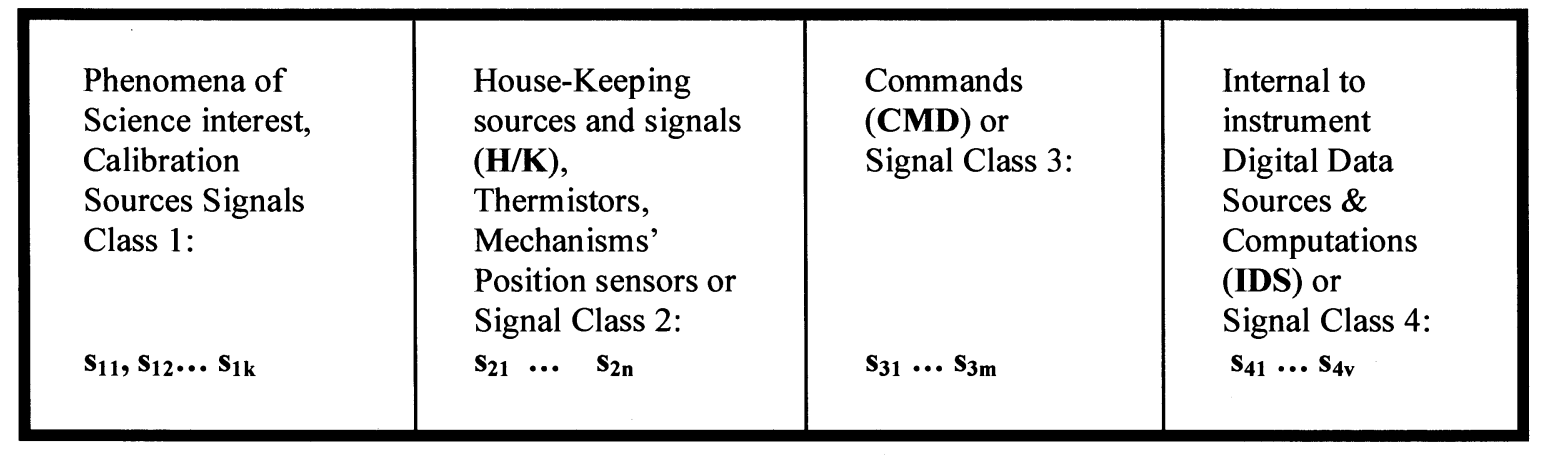

Figure 1. Classification of Instrument Analog Signals and Digital Data Sources 


\subsection{Formal Classification of Sources for Analog Signals and Digital Data}

The informal multi-classification of instrument analog signal and digital data sources in Figure 1 can be further formalized into an extended matrix as depicted in the following Figure 2. The source with most elements $(\mathrm{H} / \mathrm{K}$ is perhaps the one, because it contains dozens of thermistors) is

$$
\left\{\mathrm{s}_{21} \ldots \mathrm{s}_{2 \mathrm{n}}\right\}
$$

It comprises the class of the longest non-zero energy source of signals. Other sources for other analog signals and digital data are extended by zero-value elements to yield a sparsely populated " 4 x n" rectangular extended matrix of instrument data sources.

$\mathrm{s}_{11} \mathrm{~s}_{12} \ldots \mathrm{s}_{1 \mathrm{k}} 0_{1 \mathrm{k}+1} \ldots 0_{1 \mathrm{n}}$

$S_{21} S_{22} \ldots \quad S_{2 n-1} S_{2 n}$

$\mathrm{s}_{31} \ldots \mathrm{s}_{3 \mathrm{~m}} 0_{3 \mathrm{~m}+1} \quad \ldots \quad 0_{3 n}$

$\mathrm{s}_{41} \ldots \quad \mathrm{s}_{4 \mathrm{v}} 04 \mathrm{v}_{+1} \ldots 0_{4 \mathrm{n}}$

Figure 2. Extended Matrix of Instrument Data Sources

This formal classification matrix is used in the following Sections to derive the new instruments complexity parameters, which are further, used to develop the enhanced cost model for the electronics sub-system.

\subsection{Mapping Signal Sources Onto Instrument Detectors}

We will examine now the propagation of the signal and data elements from the formal matrix of sources (Figure 2) throughout an instrument and associated complexity parameters.

A physical phenomenon manifests itself in emanated energy from some finite size (for example, $1 \mathrm{~km} \times 1 \mathrm{~km}$ ) surface or volume region - source signal pixel and this energy is being intercepted by an instrument detector, as a sub-pixel, pixel or super-pixel (binning of a few adjacent CCD pixels, for example (Figure 3).

\subsection{On Source Signal Pixels and Detector Super-Pixels}

A Source is usually associated with some finite size geometric space, say a terrestrial 2-D surface or 3-D volume (source pixel), or a small spot on an instrument surface for mounting a thermistor - a temperature signal detector as listed in Figure 1 above. For example, consider two sources:

A ground track of dimensions $\mathrm{L} \times \mathrm{h}$ and ground pixel of size $\mathrm{h} \times \mathrm{h}$

A spot on an instrument surface of radius $r$ for mounting a thermistor 
Stylized Swath of L Source Pixels

\begin{tabular}{|l|l|l|l|}
\hline & Ground Track scanned by a spacecraft instrument telescope & \\
\hline
\end{tabular}

A circular spot on instrument surface
to mount a thermistor

\section{Figure 3. Geometry of Source Pixels}

The number $\mathbf{P}$ of source pixels for the instrument is the sum of all non-zero elements in the above matrix $\mathrm{M}$ :

$$
\mathbf{P}=\sum\left(\mathbf{s}_{\mathrm{ij}}\right)
$$

\subsection{On Mapping Signal Sources Onto Detectors and Complexity Parameters}

Instrument sensors invariably comprise a set of detectors or sensing pixels out of which a super-pixel $d_{i j}$ of size $\mathbf{Z}$ (say, $Z=3 \times 3$ ) is comprised. For example, a CCD comprises a 2-D array of $(\mathrm{n} \times \mathrm{m})$ sensing pixels that can be organized into a set of super-pixels on the CCD itself. An InGaAs linear array comprises a linear array of ( $1 \mathrm{x} \mathrm{k})$ pixels and a housekeeping thermistor sensor usually comprises a single pixel thermal detector.

Each signal source $s_{i j}$ is further defined by its measurement interval $\mathbf{t}_{\mathrm{ij}}$ in fractions of a second (or sampling rate derived from this time as its inverse with frequency in $\mathrm{Hz}$ units).

Although the sampling of different sources may not be asynchronous, the source sampling interval provides the definitive answer on the information volume of a signal source from communication point of view - in terms of source measured pixels per second.

It is obvious that at some time $\mathbf{T}$ the different samplings may intersect in time and that the instrument must be designed to handle the maximum combined data volume rate of

$$
\mathbf{P}_{\mathbf{r}}=\sum\left(\mathrm{s}_{\mathrm{ij}} / \mathrm{t}_{\mathrm{ij}}\right) \text { pixels per second for all } \mathrm{i}, \mathrm{j} \text {; the division is } 0 \text { for } \mathrm{s}_{\mathrm{ij}}=0
$$

$\mathbf{P}$ and $\mathbf{P}_{\mathbf{r}}$, the $\cdot$ volume of the instrument observed source pixels and sampling rates handled by the instrument are the two important first parameters to consider in the evaluation of the instrument complexity and cost or $\mathbf{I}_{c c}$ :

$$
\mathbf{I}_{\mathrm{cc}}=\mathbf{f}\left(\left\{\mathbf{P}, \mathbf{P}_{\mathbf{r}}\right\} \ldots\right)
$$


Next, the energy of a group of adjacent source pixels $\mathbf{s}_{\mathbf{i j}}$ energy is intercepted by an optical system and concentrated on a so-called Time Delay Integration slit of length determined by the TDI-size. This slit length $\mathbf{L}$ (in adjacent source pixels) is mapped into a TDI-size $=\mathrm{L}$ detector's spectrometer and each TDI input pixel is distributed over a column of detector $L$ super-pixels $\mathbf{d}_{\mathbf{i j}}$.

A source pixel's energy may further be practically instantaneously distributed in some spectral band over an $\lambda$-row of $\mathbf{d}_{\mathrm{ij} \lambda}$ detector super-pixels (CCD row) by different optical means, such as diffraction gratings.

This mapping of physical phenomena source pixels $s_{i j}$ onto instrument detector pixels results in the next level of data complexity, the number of detector super-pixels $\mathbf{S}$, namely

$$
\mathbf{S}=\left(\sum\left(\mathrm{d}_{\mathrm{ij}}\right)\right) / \mathbf{Z}>=\mathbf{L} \mathbf{x} \boldsymbol{\lambda}
$$

It is reasonable to evaluate the detector pixels' sampling rate by above $\mathbf{d}_{\mathrm{ij} \lambda}$ and say that

$$
\mathrm{S}_{\mathrm{r}}=\sum\left(\mathrm{d}_{\mathrm{ij \lambda}} / \mathrm{t}_{\mathrm{ij}}\right)
$$

The number $\mathbf{S}$ may be larger than $\mathbf{P}$ by an order of two magnitudes and it becomes the next important parameter in figuring out the Icc, namely:

$$
I c c=f\left(\left\{P, P_{r}, t_{i j}\right\},\left\{S, S_{r}\right\} \ldots\right)
$$

where $S>(P \times(\lambda=>60))$ or

1 source pixel is mapped onto detector $\lambda$ spectral super-pixels on one CCD row per sampling time $\mathbf{t}_{\mathrm{ij}}$.

The next level of instrument complexity stems from the detector super-pixel digitization into a fixed number of information bits $\mathbf{b}\left(\mathrm{d}_{\mathrm{ij} \lambda}\right)$. This internal to the instrument data source is the detector source super-pixel analog-to-digital signal's converter $\mathbf{A D C}_{\mathrm{ij} \lambda}$, resulting in a detector pixel digital data volume and corresponding digital data rate from analog and $\mathrm{ADC}$ board to the instrument digital information processing board or instrument data processing unit (IDPU):

$$
\begin{aligned}
& \mathbf{D}=\sum\left(\mathrm{d}_{\mathrm{ij} \lambda} \times \mathrm{b}\left(\mathrm{d}_{\mathrm{ij} \lambda}\right)\right) \\
& \mathbf{D}_{\mathbf{r}}=\sum\left(\mathrm{d}_{\mathrm{ij} \lambda} \times \mathrm{b}\left(\mathrm{d}_{\mathrm{ij} \lambda}\right) \times \mathrm{t}_{\mathrm{ij}}\right)
\end{aligned}
$$

$\mathbf{D}$ and $\mathbf{D}_{\mathbf{r}}$ are the instrument internal digital data volumes and rates that may be orders of magnitude higher than then the previous level parameters $S$ and $S_{r}$, which are related to 
analog data sources. This is because the digitization width $b\left(d_{i j \lambda}\right)=>W=>14$ bits within advanced instruments of today and tomorrow and it further contributes to Icc:

$$
\text { Icc }=\mathbf{f}\left(\left\{\mathbf{P}, \mathbf{P}_{\mathrm{r}}, \mathbf{t}_{\mathrm{ij}}\right\},\left\{\left(\mathbf{d}_{\mathrm{ij}}, \mathbf{Z}\right), \lambda, \mathbf{S}, \mathbf{S}_{\mathrm{r}}\right\},\left\{\mathbf{W}, \mathbf{D}, \mathrm{D}_{\mathrm{r}}\right\} \ldots\right)
$$

Next comes the on-board processing of the intermediate digital information within the instrument which involves transforming $\mathrm{D}, \mathrm{D}_{\mathrm{r}}$ into Intermediate data I and Intermediate rates $\boldsymbol{I}_{\boldsymbol{r}}$ that may be larger than $\mathrm{D}$ and $\mathrm{D}_{\mathrm{r}}$ by a factor of $\mathbf{L}=\mathbf{2}$ or more. For example, converting the $\mathbf{W}=\mathbf{1 4}$-bit ADC counters into a 32-bit integer or single precision floatingpoint numbers, doubles $(32 / 14=$ factor $\mathrm{L}>2)$ the volume and rate of ADC digital data to be processed within an IDPU or

$$
\begin{aligned}
I & =\mathbf{2} \times \mathbf{D} \\
I_{r} & =\mathbf{2} \times D_{r}
\end{aligned}
$$

On-board post-ADC digital data Interpolation within an instrument IDPU (required in running some on-board algorithms) and data paketization overhead may increase the data volume and rate by a factor of $\mathbf{L}>\mathbf{1 0}$. Digital domain Time Delay Integration (TDI) may, in turn, decrease the intermediate data volume and rates at the price of required computational resources to implement the TDI algorithms. This further contributes to the Icc:

$$
\text { Icc }=\mathbf{f}\left(\left\{P, P_{r}, t_{i j}\right\},\left\{d_{i j \lambda}, Z, S, S_{r}\right\},\left\{W, D, D_{r}\right\},\left\{I, I_{r}, \mathbf{L}, T \text { TDI }\right\} \ldots\right)
$$

Instrument detector calibration and fault detection may require, for example, to dump the entire CCD or an IDPU memory region. This requires the instrument to be able to handle data rates determined by native pixel-frame size and rate of full dump, say $\mathbf{F}, \mathbf{F}_{\mathbf{r}}$ or

$$
I c c=f\left(\left\{P, P_{r}, t_{i j}\right\},\left\{d_{i j \lambda}, Z, S, S_{r}\right\},\left\{W, D, D_{r}\right\},\left\{I, I_{r} ; L, T D I\right\}\left\{F, F_{r}\right\} \ldots\right)
$$

Furthermore, instrument-level data volume Reduction using Compression techniques by a pre-determined minimum factor (for example 2:1 lossless compression) may also reduce the data volume and rate to $\mathbf{R}, \mathbf{R r}$. Itbears on instrument complexity and cost:

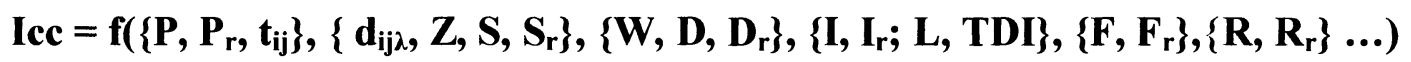

\subsection{On-Board Computational Complexity}

Following is the list of representative flight processors that demonstrates the on-board heritage computational resource:

FAST $\quad 10 \mathrm{MHz}$ general-purpose processor

SeaStar $\quad 16 \mathrm{MHz}$ a set of three primary and three backup microprocessors

TRMM $133 \mathrm{MHz}$ general-purpose processor. 
The heritage instruments, as exemplified by the instruments on spacecraft missions TRMM, SeaStar and FAST, could be characterized as

Class (I):

- High data volumes and rates

- Electronics Box is essentially through-putting high rate data to spacecraft (SC) Solid State Recorder (SSR)

- Low volume data is processed by the instrument data system computer

- These instruments are low performance software - centric and their software aspect is preeminent.

Advanced complex instruments, such as the MMS/FPI instruments require more than $\mathbf{O}=40$ Millions of Operations per second on more than $\mathbf{O}_{\mathbf{r}}=10$ Million bits per second data streams in single and double precision floating point arithmetic.

\section{Complex instruments can be characterized as RC-Hardware-Centric Data Processing Instruments or \\ Class (II):}

- High data volumes and rates

- Electronics Box is performing on-board high performance data processing in RC hardware as opposed to heritage ASIC

- Low volume data is processed by the instrument data system computer

- These instruments are high performance hardware - centric data processing instruments and their software aspect is minimal.

It is obvious from laboratory simulations that by only changing the type of variables from single precision to double precision, increases a small application's run time by a factor of. Changing data types 2 and to quad precision increases the run time by an order of magnitude. This is to simply demonstrate that on-board computations within an IDPU for an advanced instrument is not possible with heritage resources and requires FPGA implementations supplemented by host processors. In Class (II) instruments the Hardware $(\mathrm{H} / \mathrm{W})$ aspect of the Data System is the driver and the Software $(\mathrm{S} / \mathrm{W})$ aspect is minimal. This must be reflected in resource allocation, where

\section{S/W FTE $\ll$ H/W FTE}

Because of this the computational complexity parameters $\mathbf{O}$ and $\mathbf{O}_{\mathbf{r}}\left(\right.$ where often $\left.\mathbf{O}_{\mathbf{r}}=\mathbf{D}_{\mathbf{r}}\right)$ must be included in the instrument IDPU conceptual design and cost model:

$$
\begin{gathered}
I c c=f\left(\left\{P, P_{r}, t_{i j}\right\},\left\{d_{i j \lambda}, Z, S_{,}, S_{r}\right\},\left\{W, D, D_{r}\right\},\left\{I, I_{r} ; L, T D I\right\},\left\{F, F_{r}\right\},\right. \\
\left.\left\{R, R_{r}\right\},\left\{O, O_{r}\right\}\right)
\end{gathered}
$$




\subsection{Instrument Flight Software for a Constellation}

The Instrument Flight Software f or Firmware - be to processor software or FPGA VHDL Code significantly affects the cost model. The flight software complexity affecting the model is designated as $\mathbf{f}$ or $\mathbf{f} / \mathbf{m}$, where $\mathbf{m}$ is the number of homogeneous spacecrafts/instruments in the constellation configured with the same instrument software with the $\mathrm{S} / \mathrm{W}$ development costs counted only once. The software size $\mathbf{f}$ also bears on model cost. The cost model then includes the associated S/W parameter f:

$$
\begin{gathered}
I c c=\mathbf{f}\left(\left\{\mathbf{P}, \mathbf{P}_{\mathbf{r}}, \mathbf{t}_{\mathrm{ij}}\right\},\left\{\mathbf{d}_{\mathrm{ij} \lambda}, \mathbf{Z}, \mathbf{S}, \mathbf{S}_{\mathbf{r}}\right\},\left\{\mathbf{W}, \mathbf{D}, \mathbf{D}_{\mathbf{r}}\right\},\left\{I, I_{\mathbf{r}} ; \mathbf{L}, \mathbf{T D I}\right\},\left\{\mathbf{F}, \mathbf{F}_{\mathbf{r}}\right\},\right. \\
\left.\left\{\mathbf{R}, \mathbf{R}_{\mathbf{r}}\right\},\left\{\mathbf{O}, \mathbf{O}_{\mathbf{r}}\right\}, \mathbf{f}\right)
\end{gathered}
$$

\subsection{Computation Arithmetic}

Furthermore, the choice of arithmetic $\mathbf{A}$ - floating-point as opposed to fixed-point arithmetic affects

\section{Input Data Dynamic Range}

Allowable Depth of Computations before loosing precision

Processing Time Depth.

In other words, the arithmetic choice parameter bears significant design costs, increasing with fixed-point, in particular and reflecting in the cost model:

$$
\begin{gathered}
I c c=f\left(\left\{P, P_{r}, t_{i j}\right\},\left\{d_{i j \lambda}, Z, S, S_{r}\right\},\left\{W, D, D_{r}\right\},\left\{I, I_{r} ; L, T D I\right\},\left\{F, F_{r}\right\},\right. \\
\left.\left\{R, R_{r}\right\},\left\{O, O_{r}\right\},\{f\},\left\{A, A_{r}\right\}\right)
\end{gathered}
$$

\subsection{Calibration Sources Complexity}

And finally, the calibration sources complexity must be taken into account by introducing parameter $\mathbf{M}$ for "Mimicking science phenomena" data sources. This completes the new enhanced cost model framework:

$$
\begin{gathered}
I c c=f\left(\left\{P, P_{r}, t_{i j}\right\},\left\{d_{i j \lambda}, Z, S, S_{r}\right\},\left\{W, D, D_{r}\right\},\left\{I, I_{r} ; L, T D I\right\},\left\{F, F_{r}\right\},\right. \\
\left.\left\{R, R_{r}\right\},\left\{O, O_{r}\right\},\{f\},\left\{A, A_{r}\right\}, M\right)
\end{gathered}
$$

\subsection{Enhanced Cost Model Using Complexity Index}

The heritage instrument performance model in use for heritage instrument cost evaluation is based on throughput parameters $\mathbf{P}, \mathbf{D}, \mathbf{P}_{\mathbf{r}}, \mathbf{D}_{\mathbf{r}}$ and heritage hardware designs $\mathbf{H}$ :

$$
\begin{aligned}
& \mathbf{v}=\mathbf{v}(\mathbf{P}, \mathbf{D}) \\
& \mathbf{v}_{\mathbf{r}}=\mathbf{v}_{\mathbf{r}}\left(\mathbf{P}_{\mathbf{r}}, \mathbf{D}_{\mathbf{r}}\right) \\
& \mathbf{u}=\mathbf{u}\left(\mathbf{v}, \mathbf{v}_{\mathbf{r}}, \mathbf{H}\right)=\mathbf{u}\left(\mathbf{P}, \mathbf{D}, \mathbf{P}_{\mathbf{r}}, D_{\mathbf{r}}, H\right)
\end{aligned}
$$

A complex instrument data system must be designed to handle the largest data volume and data rates determined by all parameters and by employing new solutions in 
reconfigurable hardware (RC). The enhanced cost model depends on the system complexity index $\mathrm{K}$ derived from the complexity parameters:

$$
\begin{aligned}
& \mathbf{V}=\max (\mathbf{P}, \mathbf{S}, \mathbf{D}, \mathbf{I}, \mathbf{F}, \mathbf{R}, \mathbf{O}, \mathbf{f}, \mathbf{A}, \mathbf{M}) \\
& \mathbf{V}_{\mathbf{r}}=\max \left(\mathbf{P}_{\mathbf{r}}, \mathbf{S}_{\mathbf{r}}, \mathbf{D}_{\mathbf{r}}, \mathbf{I}_{\mathbf{r}}, \mathbf{F}_{\mathbf{r}}, \mathbf{R}_{\mathbf{r}}, \mathbf{O}_{\mathbf{r}}, \mathbf{A}_{\mathbf{r}}, \mathbf{M}_{\mathbf{r}}\right) \\
& \mathbf{U}=\mathbf{U}\left(\mathbf{V}, \mathbf{V}_{\mathbf{r}}, \mathbf{R C}, \mathbf{K}\right)=\mathbf{U}(\mathbf{P}, \mathbf{S}, \mathbf{D}, \mathbf{I}, \mathbf{F}, \mathbf{R}, \mathbf{O}, \mathbf{f}, \mathbf{A}, \mathbf{M}, \\
&\left.\mathbf{P}_{\mathbf{r}}, \mathbf{S}_{\mathbf{r}}, \mathbf{D}_{\mathbf{r}}, \mathbf{I}_{\mathbf{r}}, \mathbf{F}_{\mathbf{r}}, \mathbf{R}_{\mathbf{r}}, \mathbf{O}_{\mathbf{r}}, \mathbf{A}_{\mathbf{r}}, \mathbf{M}_{\mathbf{r}}, \mathbf{R C}\right)
\end{aligned}
$$

It is not that the new cost model parameters $\mathrm{S}, \mathrm{I}, \mathrm{F}, \mathrm{R}, \mathrm{O}, \mathrm{f}, \mathrm{A}, \mathrm{M}$ and their corresponding rate parameters $\mathbf{S}_{\mathbf{r}}, \mathbf{I}_{\mathbf{r}}, \mathbf{F}_{\mathbf{r}}, \mathbf{R}_{\mathbf{r}}, \mathbf{O}_{\mathbf{r}}, \mathbf{A}_{\mathbf{r}}, \mathbf{M}_{\mathbf{r}}$ were not in the considerations before. However, their affects were so negligent in comparison with $P$ and $D$ parameters, that they could be reasonably ignored at conceptual level design. When they were considerable, the heritage ASICs implementation came to the rescue sometimes accompanied by re-designs, at a significant cost at depicted in the following Figure 5.

\subsection{Empirical Evaluation of the Enhanced Cost Model}

If heritage cost model $\mathrm{u}=\mathrm{u}\left(\mathrm{P}, \mathrm{P}_{\mathrm{r}}, \mathrm{D}, \mathrm{D}_{\mathrm{r}}, \mathrm{H}\right)$ resulting cost was $\mathrm{u}=5 * \$ \mathrm{X}$ (in some heritage cost unit, say millions of dollars) or $\$ 5 \mathrm{X}$, where 5 was the number of parameters in the heritage cost model, namely the parameters $\mathrm{P}, \mathrm{D}, \mathrm{P}_{\mathrm{r}}, \mathrm{D}_{\mathrm{r}}$. The enhanced cost model empirical evaluation can be represented as

$$
\begin{gathered}
\mathbf{U}=\mathbf{U}\left(\mathbf{V}, \mathbf{V}_{\mathbf{r}}, \mathbf{R C}, \mathbf{K}\right) \sim \mathrm{u}\left(\mathrm{v}, \mathrm{v}_{\mathrm{r}}, \mathrm{H}\right)+\mathbf{K}\left(\mathbf{V}, \mathbf{V}_{\mathbf{r}}\right) \text { or } \\
\mathbf{U}=\mathbf{u}+\mathbf{S k}=\mathbf{u}+(\mathbf{\$ 4 . 0} \text { million dollars })
\end{gathered}
$$

The cost adjustment parameter $\mathbf{k}$ is an empirical evaluation of the complexity index

where $\mathbf{k}$ was derived as

$$
K=K\left(V, V_{r}\right) \sim k
$$

$$
\mathbf{K}\left(\mathbf{V}, \mathbf{V}_{\mathbf{r}}\right) \sim \mathbf{k}=\left(\left(\|\mathbf{V}\|+\left\|\mathbf{V}_{\mathbf{r}}\right\|\right) /\left(\|\mathbf{v}\|+\left\|\mathbf{V}_{\mathbf{r}}\right\|\right)\right)
$$

In this paper $\mathbf{k}=(20 / 5)=4.0$, where 20 is the number of complexity parameters in the enhanced cost model and 5 is the number of parameters in the heritage cost model. Factor $\mathbf{k}$ is also based on the experience gleaned from the development of a few recent missions, and expressed as the heritage cost model adjustment, in $\mathbf{k}$ millions of dollars $(\$ \mathbf{k M})$. Factor $\mathbf{k}$ can be refined in future work as complexity index $K=K\left(\mathbf{V}, \mathbf{V}_{\mathbf{r}}\right)$ gets a better formulation and by design optimization outlined below in Section 8 .

The sequence of these complexity parameters are the nodes on the guiding line from the heritage cost model $\boldsymbol{u}$ to the new enhanced cost model $\boldsymbol{U}$ :

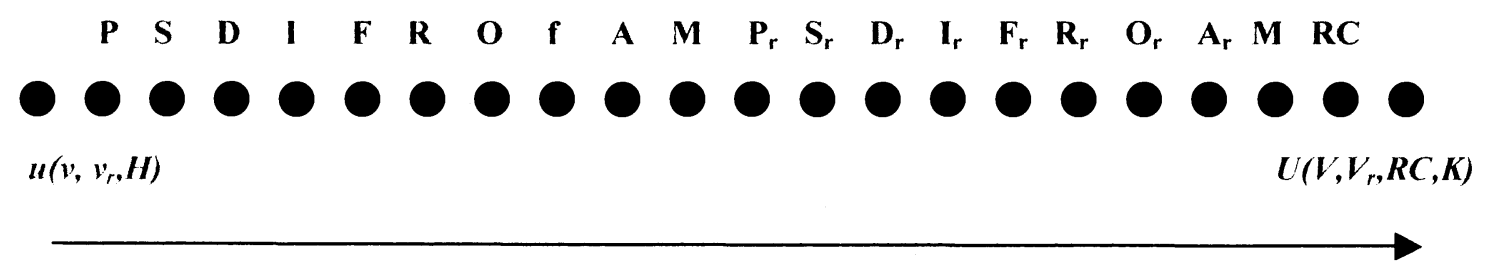

Figure 4. Guiding Line From Heritage Cost Model $u$ to the Enhanced Cost Model $U$ 


\subsection{Enhanced Cost Model Diagram}

The enhanced cost model dependencies diagram present time inputs to the model and its functionality, as well as all the complexity parameters enumerated in this paper.

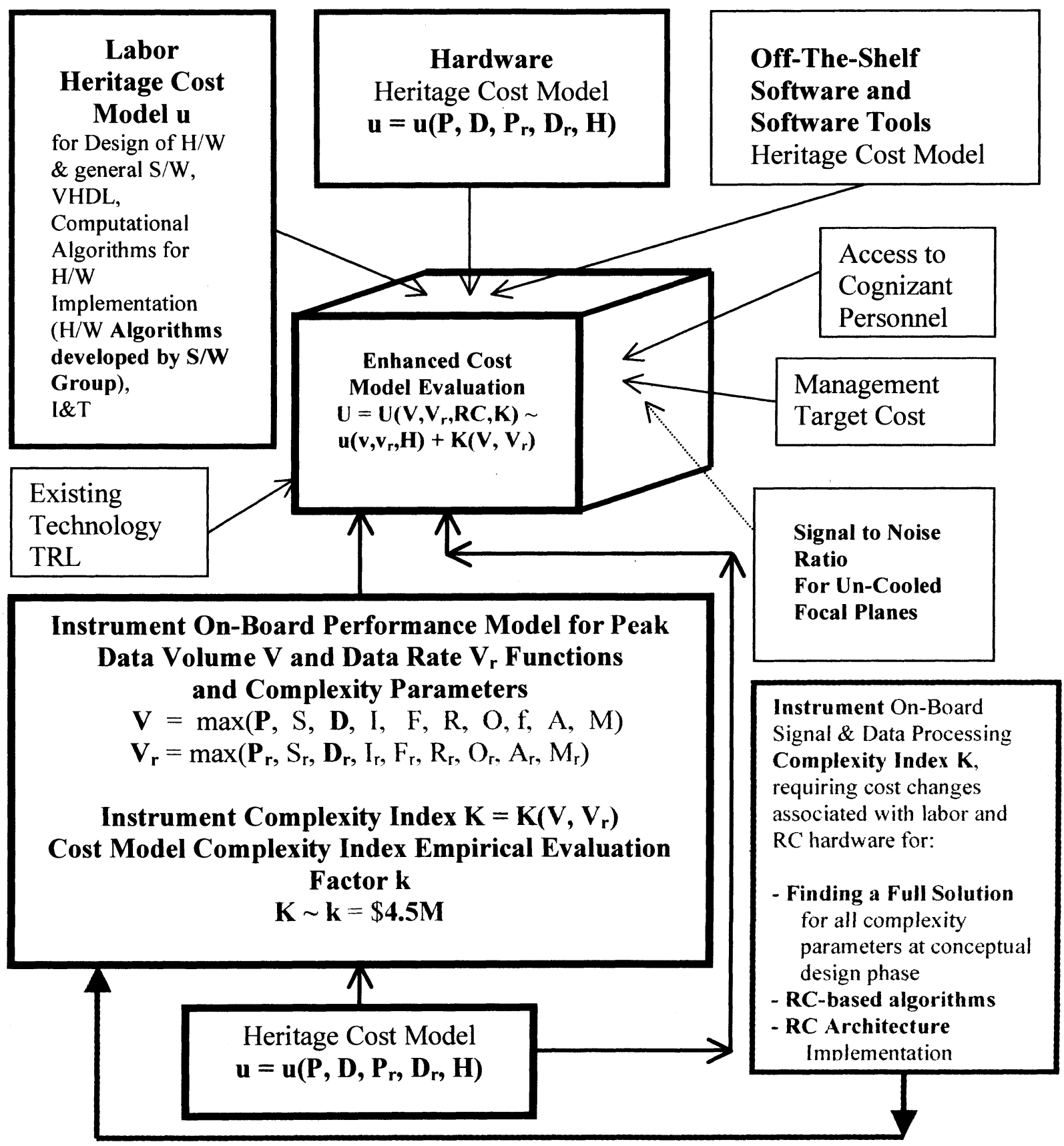

Figure 5. Enhanced Cost Model U Dependencies 


\subsection{Design of the EE Subsystem to Complexity Parameters and Options}

The cost caps for contemporary complex instruments discourage ASIC and cryogenicsbased solutions (Figure 6). The instruments of today and tomorrow are characterized by complexity parameters that are larger than $\mathbf{P}$ and $\mathbf{D}$ by orders of magnitude, resulting in the need to reconsider the IDPU Complexity and Cost Models more explicitly. The resulting enhanced cost model is very different from the heritage cost model, as depicted in the Enhanced Cost Model Diagram in Figure 5. The new solutions for complex instruments are based on FPGA/RC technology implementations, which also allow solutions for a larger class of problems, but at a lower cost than with ASICs. Following are some implementation options for complex instruments depicted in a pictorial form.

\subsection{Implementation Options}

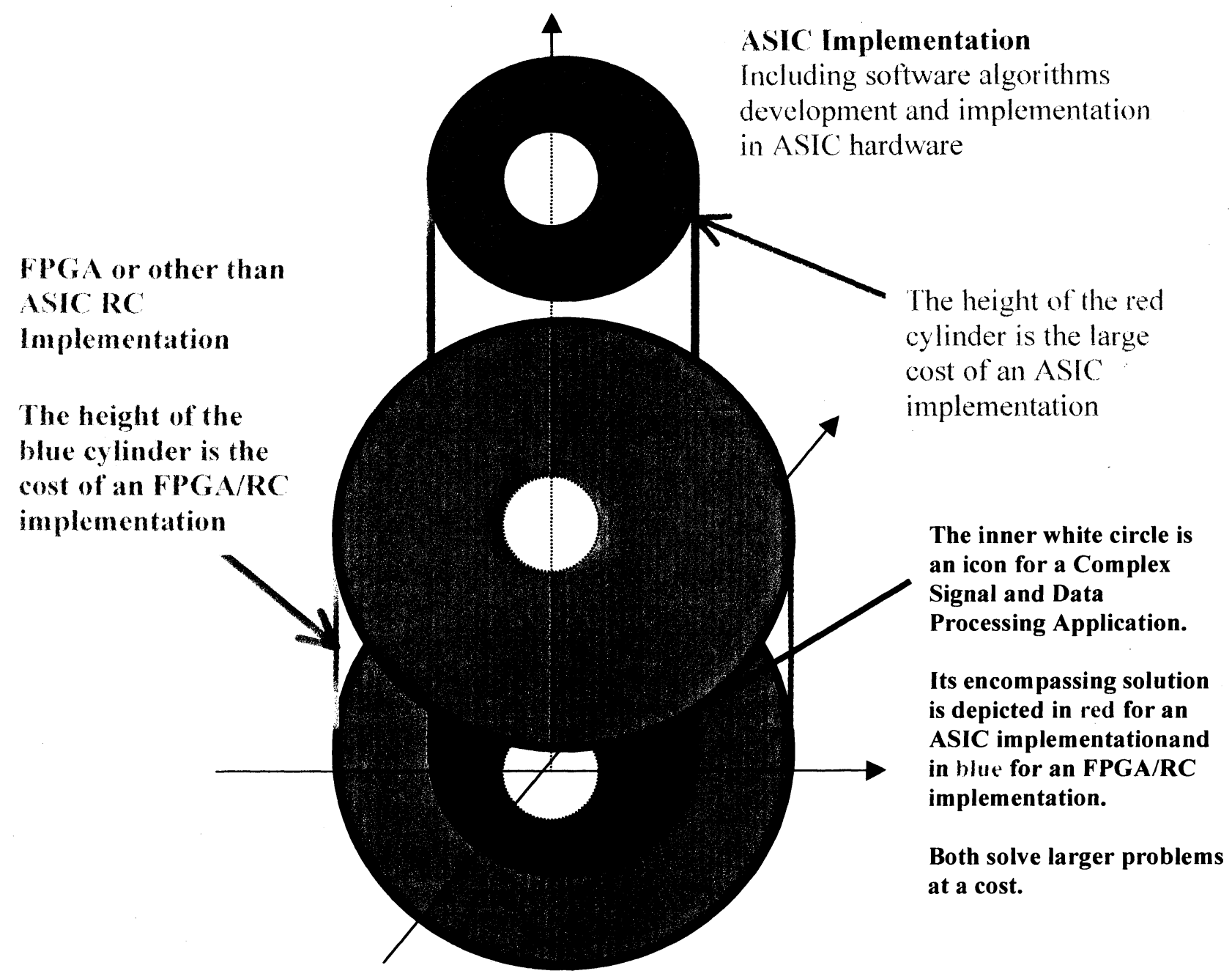

Figure 5. Comparative Costs of ASIC and FPGA Solution Implementations 


\subsection{Design of an EE Subsystem to Complex Parameters}

Contemporary complex instruments and their electrical and electronics data sub-systems must be designed from the conceptual outset to their complexity parameters and include these performance complexity parameters in the enhanced cost model $\mathbf{U}$. The design to complexity parameters and its optimization may greatly affect the cost associated with the instrument complexity index $\mathbf{K}$, or even change its sign, resulting in overall cost reduction. For instance, an improvement of an on-board computational algorithm by a factor of 2 is huge because of the cost of on-board resources, while on the ground it is not worth the effort to achieve it. The design and optimization of an instrument on-board data system can, in turn, significantly affect the value and sign of the new cost model for the complexity index $K$, reducing costs in other parts of the mission, like downlink bandwidth and ground processing.

For example, in instruments dealing with weak measurement signals, an on-board digital TDI computation is an algorithm of choice. The n-step TDI (TDI-n) algorithm is critical in improving the signal-to-noise ratio for weak Near Infrared (IR) and thermal band signals by a factor of $\operatorname{sqrt}(\mathbf{n})$. As a byproduct of TDI-n process, the downlink data volume is decremented by a factor of $\mathbf{n}$. However, while source signal noise randomness accounts for averaging it out the analog signal digitization noise only adds up in lower bits of the digital counts and the lower two bits in the TDI output need to be discarded. The design to the intermediate parameter $\boldsymbol{I}$ requires that the ADC is 2-bits wider than toplevel requirements for the width of the downlink counters. On the other hand, the TDI computation of complexity $\boldsymbol{O}$ on this larger volume of intermediate data can be optimized by a factor of at least 2 by replacing the operation of division with multiplication by a pre-computed and stored in a small table of multiplicands $1 / \mathrm{j}$, for $1<=\mathrm{j}<=24$.

In any contemporary implementation of the operation of division the division takes 15-25 clock cycles, as compared to 3 cycles for subtraction, addition, and multiplication. When there is a short computation (like a TDI-6), involving five additions $(+)$, and a single division (/), the division is much more lengthy in time cycles' duration than an entire TDI computation $\left(a_{1}+a_{2}+a_{3}+a_{4}+a_{5}+a_{6}\right) / 6$, where $d^{j}$ are the detector pixels signal measurements for ground pixel $\boldsymbol{a}$ at consecutive dwelling times $t_{j}$.

However, when the dividers are known in advance and their number is small, for example in a TDI- $j$ computation $(1<=j<=24)$, their inverse can be pre-computed and stored in a small table. Then, instead of division implementation in FPGA or its computer use, can be replaced by multiplication making the entire computation at least twice faster and coherent without any additional resources for an FPGA divider circuitry.

The design and development of the electronics data subsystem, based on the above derived complexity parameters, requires personnel labor and reconfigurable hardware, commensurate with the resulting correction factor $\boldsymbol{k}$. Namely, it is empirically known that each million of dollars in cost is equivalent to 3 Full Time Equivalent developer labor 
units (FTEs). The cost attributed to complexity index $K=5$ can be approximated by $(3 * \boldsymbol{k})$ FTES (approximately 15 FTES) over the mission development period, in addition to the heritage cost which basically covers the materials, manufacturing and processor software, rather than the design to the complex parameters.

\section{Conclusions}

We characterized the class of representative complex instruments as Class II of RChardware-centric data processing instruments. In such instruments the complexity of on-board analog signal and digital data processing in reconfigurable hardware far supersedes that in the general-purpose heritage processors. For such instruments the EE subsystem development effort supersedes the heritage software-centric development methodology and an enhanced cost model must be applied to it. We have then analyzed the new representative complex instrument top-level requirements and associated data volumes, rates and on-board computational algorithms' RC implementations. We enumerated the EE subsystem data processing complexity parameters and functions $\mathbf{V}$, $\mathbf{V}_{\mathbf{r}}$ and introduced the instrument complexity index $\mathbf{K}\left(\mathbf{V}, \mathbf{V}_{\mathbf{r}}\right)$. We then introduced the complex instrument EE subsystem enhanced cost model framework $U\left(V, V_{r}, R C, K\right)$. Cost Model $\mathbf{U}$ is based on labor required to design for the complexity parameters and on cost of reconfigurable hardware. For complex instruments the EE subsystem must be designed to the complexity parameters one-by-one at the outset conceptual level. The costing must use the enhanced cost model $\mathbf{U}$ that is based on the complexity parameters in order to avoid consecutive development costs' diversions and schedule overruns. The costs associated with the complexity parameters can be estimated in heritage ways, as long as the complexity parameters are costed at the outset conceptual level design and converted to labor and $\mathrm{RC}$ hardware inputs used by a heritage cost model. We derived the empirical evaluation of the enhanced cost model function as $\mathbf{U} \sim \mathbf{u}+\mathbf{k}(\mathbf{K})$. Factor $\mathbf{k}$ can also be viewed as the baseline cost of a complex instrument EE subsystem data. Future work consists in implementing the enhanced cost model framework into a cost engineering tool.

\section{Acknowledgements}

We would like to thank Paul Earle for constructive discussions of the paper.

\section{References}

[1] Model-H, Personal Communication from Paul Earle/NASA GSFC

[2] Cost Model for Electronics Architecture Trade Studies by Jakob Axelsson, Volvo Technological Corp., 2000 IEEE

[3] Parametric Cost Estimating by Ralph W. Farrington, RWF Consulting LLC, 2005 IEEE. 


\section{Biographies}

Semion Kizhner is an aerospace engineer with the National Aeronautics and Space Administration at the Goddard Space Flight Center. He proposed the development of the Hilbert-Huang Transform Data Processing System and has been leading the HHTDPS development team. He participated recently in evaluation of the NASA Advanced Space Technology proposals. He published a dozen of technical papers and mentored numerous undergraduate, graduate and doctoral students in the NASA Education Programs. He graduated from Johns Hopkins University with an MS degree in computer science.

Umesh D. Patel received his D.Sc. degree from the George Washington University, Washington, DC. Since 1995, he has been working at Goddard Space Flight Center, Greenbelt, MD as Electronics Engineer. He has authored and co-authored papers in technical journals and conferences. His research interests are hysteresis modeling, lowvoltage mixed-signal integrated circuit designs, radiation tolerant microelectronics and data conversion VLSI systems designs.

Robert Kasa is an electrical engineering manager at the NASA Goddard Space Flight Center. He has a Bachelor of Science degree in Engineering from Purdue University, and has 24 years of aerospace experience. He has directed integration and test programs and has led flight electronics development teams such as the Space Shuttle Hitchhiker Space Flight Carrier and the BBXRT/ASTRO-1 Missions. As Head of Goddard's Microelectronics and Signal Processing Branch since 1997, Mr. Kasa has been responsible for the electronics development for several major scientific spaceflight instruments, for both Earth orbit and visits to the inner and outer planets.

Phyllis Hestnes is an electrical engineer at NASA Goddard Space Flight Center. She graduated from the University of Maryland. Her primary interests include the design of processor based systems and reconfigurable computing. She is presently the Associate Branch Head of the NASA Goddard Microelectronics and Signal Processing Branch.

Tammy Brown is the Team Lead of the Instrument Design Lab (IDL) for the Integrated Design Center (IDC) at NASA Goddard Space Flight Center. The IDL provides concurrent, collaborative engineering support to create conceptual instrument designs for feasibility studies and strategic mission planning. She holds a B.S. degree in electrical engineering from the University of Pittsburgh, and has worked at Goddard in remote sensing applications for over 15 years.

Madhavi 'Meg' Vootukuru is a founder of Syneren Technologies Corporation, a company providing high quality engineering and program support services to DoD and other federal agencies as well as commercial customers. She graduated from University of Cincinnati, with a Master of Science in Electrical and Computer Engineering. She has over 10 years of industry experience in the fields of ASIC/FPGA/Microprocessor Design and Verification with companies like Digital Equipment Corporation and Motorola. She was a key contributor in the Alpha Microprocessor Design while at Digital and worked on Network Processors at Motorola. She has authored and reviewed several technical papers in conferences and Journals. 